(PUBLISHER-WILEY, USA)

Accepted $15^{\text {th }}$ November 2020

Editor: Dr. William M. Worek (TEXAS, USA)

Online ISSN:2688-4542

\title{
"MIXED CONVECTION FLOWS OF TANGENT HYPERBOLIC FLUID PAST AN ISOTHERMAL WEDGE WITH ENTROPY: A MATHEMATICAL STUDY"
}

P. Ramesh Reddy1, S. Abdul Gaffar2, B. Md. Hidayathulla Khan 3 , K. Venkatadri4, O. Anwar Beg 5 ${ }_{1}$ Department of Mathematics, Madanapalle Institute of Technology and Science, Madanapalle, India 2Department of Information Technology, Mathematics Section, University of Technology \& Applied Sciences, Salalah, Oman ${ }_{3}$ Department of Mathematics, Aditya college of Engineering, Madanapalle, India

${ }_{4}$ Department of Mathematics, Sreenivasa Institute of Technology and management studies, Chittoor-517127, India 5Magnetohydrodynamics, Biological Propulsion and Energy Research, Aeronautical and Mechanical Engineering Division, University of Salford, M5 4WT, UK. 


\section{MIXED CONVECTION FLOWS OF TANGENT HYPERBOLIC FLUID PAST AN ISOTHERMAL WEDGE WITH ENTROPY: A MATHEMATICAL STUDY

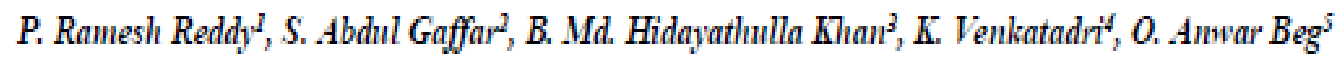 \\ 'Department of Mathematics, Madanapalle Institute of Tochnology and Science, Madanapalle, India \\ ${ }^{2}$ Department of Information Technology, Mathematics Section, University of Tochnology \& Appliad Sciences, Salalah, Oman \\ ${ }^{3}$ Department of Mathematics, Aditya colloge of Engineering, Madanapalle, India \\ ${ }^{4}$ Department of Mathematics, Sreenivasa Institute of Tochnology and management studies, Chittoor-517127, India \\ ${ }^{5}$ Magnetohydrodynamics, Biological Propulsion and Energy Research, Aeronautical and Mochanical Engineering Division, University of Salford, MS $4 \pi T, U K$}

\section{ABSTRACT}

The non-linear, steady, mixed convective boundary layer flow and heat transfer of an incompressible tangent hyperbolic non-Newtonian fluid from an isothermal wedge in the presence of magnetic field is analyzed numerically using the Keller Box implicit finite difference technique. The entropy analysis due to MHD flow of tangent hyperbolic fluid past an isothermal wedge and viscous dissipation is also included. The numerical code is validated with previous Newtonian studies available in the literature. Graphical and tabulated results are analyzed to study the behavior of fluid velocity, temperature, concentration, shear stress, heat transfer rate, entropy generation number and Bejan number for various emerging thermo physical parameters, namely, Weissenberg number $(W e)$, power law index $(n)$, mixed convection parameter $(\lambda)$, pressure gradient parameter $(m)$, Prandtl number $(\mathrm{Pr})$, Biot number $(\gamma)$, Hartmann number $(\mathrm{Ha})$, Brinkmann number $(\mathrm{Br})$, Reynolds number $(R e)$ and temperature gradient $(I I)$. It is observed that velocity, entropy, Bejan number and surface heat transfer rate are reduced with increasing Weissenberg mumber, but temperature and local skin friction are increased. Increasing pressure gradient enhances velocity, entropy, local skin friction and surface heat transfer rate but reduces temperature and Bejan number. An increase in an isothermal power law index (n) is observed to increase velocity, Bejan number and surface heat transfer rate but decreases temperature, entropy and local skin friction. Increasing magnetic parameter $(\mathrm{Ha})$ is found to decrease temperature, entropy, surface heat transfer rate and local skin friction and increases velocity and Bejan number. The research is applicable for coating materials in chemical engineering for instance, robust paints, production of aerosol deposition and water sohuble solution thermal treatment. 
KEYWORDS: Tangent Hyperbolic fluid; Weissenberg mamber; power law index; Hartmann mumber; Mixed Convection; pressure gradient parameter; ...

Author for Correspondence: Email: abdulsgaffar0905@gmail.com

NOMENCLATURE

\begin{tabular}{|c|c|c|c|}
\hline$B_{0}$ & externally imposed radial magnetic field & & Greek Symbols \\
\hline $\mathrm{Be}$ & Dimensionless Bejan mumber & $\alpha$ & Thermal diffusivity \\
\hline $\mathrm{Br}$ & Brinkmann mumber & $\beta$ & The coefficient of thermal expansion \\
\hline$C$ & Positive constant & $\Omega$ & Wedge angle \\
\hline$C_{f}$ & Skin Friction Coefficient & $\sigma$ & Electric conductivity of the fluid \\
\hline$f$ & Dimensionless stream fimction & $\eta$ & Non-dimensional radial coordinate \\
\hline$g$ & Gravitational acceleration & $\mu$ & Dynamic viscosity \\
\hline$G \gamma_{x}$ & Local Grashof mumber & $\xi$ & Non-dimensional tangential coordinate \\
\hline $\mathrm{Ha}$ & Hartmann mumber (magnetic parameter) & $\psi$ & Non-dimensional stream function \\
\hline$h_{w}$ & convective heat transfer coefficient & $v$ & Kinematic viscosity \\
\hline$k$ & Thermal conductivity of the fluid & $\Gamma$ & Time dependent material constant \\
\hline$m$ & Pressure gradient & $\theta$ & Dimensionless temperature \\
\hline$n$ & Power law index i.e. flow behavior index & $\rho$ & Fluid density \\
\hline $\mathrm{Ng}$ & Entropy generation mumber & $\Pi$ & Second invariant strain tensor \\
\hline $\mathrm{Nu}$ & Heat transfer coefficient & $\mu_{\infty}$ & infinite shear rate viscosity \\
\hline $\operatorname{Pr}$ & Prandtl mumber & $\gamma$ & Biot number \\
\hline $\operatorname{Rex}_{x}$ & Reynolds Number & $\overline{\bar{\tau}}$ & extra stress tensor \\
\hline$T$ & Fluid temperature & $\Pi_{l}$ & Temperature gradient \\
\hline$\overline{T_{w}}$ & Convective fluid temperature & $\beta_{1}$ & Total angle of the wedge \\
\hline$U_{\infty}$ & Free steam velocity & $\lambda$ & Mixed convection parameter \\
\hline$u, v$ & $\begin{array}{l}\text { Dimensionless velocity components in } X \text { and } \\
Y \text { direction respectively }\end{array}$ & $\mu_{0}$ & zero shear rate viscosity \\
\hline$V$ & Velocity vector & $\bar{\gamma}$ & shear rate \\
\hline We & Local Weissenberg mumber & & Subscripts \\
\hline$x$ & Stream wise coordinate & $w$ & Conditions on the wall \\
\hline$y$ & Transverse coordinate & $\infty$ & Free stream condition \\
\hline
\end{tabular}




\section{INTRODUCTION}

Due to constantly new advances in technology and engineering, non-Newtonian liquids became an issue of considerable interest to researchers for over past few decades. Sources of these fluids comprise coal-oil slurries, detergents, pain, smart coatings and suspension, grease. Cosmetics, custard, biological liquids, etc. The non-Newtonian fluids constitutive equations can never be characterized with one constitutive relation involving rate of strain and stress like the viscous (Newtonian) fluids. These fluids have complex attributes in nature and industry. Generally, the mathematical model of the non-Newtonian liquids is complex due to the significant nonlinearity and higher order differential transportation equations especially as compared to viscous fluids. Considering their complications, researchers are involved in dynamics of non-Newtonian liquids as such fluids have wide applications in biotechnological production, plastic manufacturing, medical sciences, etc. Though the system of equations evolving in non-Newtonian fluids typically need access to numerical techniques, in some restricted cases closed form analytical solutions were achieved. Fetecau et al. [1] presented the Caputo-Fabrizio's concept of nonlinear systems to obtain appropriate sohution for second-grade hydromagnetic movement of fluids past a moving infinite flat plate. Geethan et al. [2] considered the 3D hydromagnetic chemically radiative flows of Jeffery's fluid past the stretchable porous surface considering the effects of heat source/sink and Soret number. Shehzad et al. [3] under the implications of thermophoresis and the Cattaneo-Christov principle conducted the numerical study of Maxwell fluid flows past revolving disk. Panchun et al. [4] studied the rotational electro-osmotic flow of Oldroyd-B fluid in a microchannel. They also considered the Oldroyd-B fluid's viscoelasticity and slip effect on electroosmotic flow velocity distributions. Ijaz et al. [5] discussed the Carreau fluid's stagnation point MHD flows past a stretching and shrinking cylindrical surface. Meenakumari and Lakshminarayana [6] employed shooting technique to investigate the unsteady chemically radiative MHD 3D Williamson fluid flow past a stretching surface considering the effects of Hall current and heat source/sink. Sayer et al. [7] discussed the entropy generation of MHD thermally radiative Eyring-Powell fluid through an unstable oscillating porous stretchable surface. Das et al. [8] examined the impact of Soret and variable heat absorption on unsteady chemically radiative MHD flows of Casson fluid through a stretchable porous surface. Chamkha et al. [9] investigated the entropy generation of MHD mixed convective nanofluid flows in a Gamma shaped porous cavity. 
The Tangent Hyperbolic fluid is a four-constant fluid model, designed to explain the shear thinning characteristics, is amongst the non-Newtonian constitutive models that have arisen [10] - [11]. This Tangent Hyperbolic fluid model has many applications in chemical engineering and it does have few benefits over many various non-Newtonian liquid models. It has be observed in lab experiments that the present model accurately describes the blood flows. The tangent hyperbolic fluid is a rate type fluid model that describes the attributes of relaxation and the retardation times. This model represents the viscosity depending on shear rate as a mathematical hyperbolic tangent function The apparent viscosity of such fluids steadily varies from zero shear rate to infinity shear rate. Few prime examples of such fluid include, magma, ketchup, whipped cream, blood, sprays coating and so on. Using the hyperbolic tangent model, calendaring, which is a continuous production activity for thermoplastic sheets or films of uniform thickness accomplished via a pair of heated driven rolls of equal or unequal diameters, was simulated by Kiparissides and Vlachopoulos [12]. Magnetohydrodynamics abbreviated as MHD, is the study of extremely electrically conductive fluids with magnetic characteristics. The electrically induced fluid flows all over the magnetic field and current generates and hence the flow and the magnetic field effects variate. In various fields such as oil and natural gases, atmospheric sciences, agricultural production, geosciences, solar and astro physics, MHD plays a leading role. Examples of magento liquids inclue electrolytes, sea water, oil metals and plasma. Zakir et al. [10] presented the Lie group analysis of an incompressible tangent-hyperbolic fluid MHD convection considering the velocity and thermal slip effects along with suction/injection effects. Oyelakin and P. Sibanda [13] analyzed the importance of variable viscosity and thermal conductivity in tangent-hyperbolic MHD flows. Veera and Chamkha [14] addressed the impacts of Dufor, radiation and heat generation on MHD convection Nanofluid flows past a semi-infinite porous plate considering the Hall and Ionslip effects. Kumar et al [15] examined the MHD mixed convection flows of optically thin fluid past a vertical plate considering the radiation and dissipation effects. Mamata et al. [16] employed the shooting technique to explore the impacts of heat source on chemically radiative MHD flows of tangent-hyperbolic liquid past an exponentially expanding sheet. Faroog et al. [17] examined the slip and chemical reaction effects on peristalsis of tangent hyperbolic fluid in a curved channel. Ganesh et al. [18] proposed the thermally radiative MHD flows of tangent hyperbolic fluid over a stretching sheet with suspension of the fluid particles. Jitendra et al. [19] investigates the effects of Hall current on MHD mixed convection flows of viscoelastic fluid past 
vertical surface using the perturbation technique. Venkatadri et al. [21] theoretically presented the radiative MHD nanofluid flows across a progressively stretching/shrinking permeable surface with melting. Aziz et al. [21] presented the rheological characteristics of 3D couple stress chemically radiative nanofluid MHD flows induced by oscillating stretchable surface.

Falkner-Skan flows [22] have been considered by very few researchers. The 2D wedge design is connected with this family of boundary layer flows. Non-Newtonian flows past wedge geometries emerge in various chemical engineering fields using the second-order Reiner-Rivlin model. A significant attention has been drawn towards the mixed convection boundary layer flux past a wedge. In the presence of thermal radiation with a Rosseland diffusion approximation, a non-similar solution of mixed convection flow of a Nanofluid past an isothermal wedge immersed in a permeable porous medium was examined by Chamkha et al. [23]. Takhar et al. [24] discussed in the presence of magnetic field, the unsteady mixed convection flow over a rotating vertical cone. Gorla et al. [25] analyzed the mixed convection boundary layer flows of Nanofluid past a vertical wedge immersed in a permeable porous medium Thameem ot al. [26] reported the forced convection Falkner-Skan flow of a single walled carbon NanoHorn and water Nanofluid past a wedge, plate and stagnation point in the presence of nonlinear radiation and induced magnetic field. Chamkha et al. [27] explored the radiative mixed convection boundary layer flow of Nanofluid past a vertical cone immersed in a porous media.

Convective heat transfer has generated significant attention due to its significance in energy and biological innovations like power generation, diesel generators, nuclear power plants, missile technology, hydroelectric reserves, photovoltaic systems etc. Also, the convective boundary condition has fascinated considerable attention and is typically synchronized in the thermal boundary condition of the wall via a Biot number. Nurul et al. [28] examined the MHD mixed convection stagnation point flow of hybrid nanofluid through a vertical flat plate with convective boundary condition. Hayat et al. [29] dealt with the impact of binary chemical reaction and activation energy in third grade nanofluid hydromagnetic flow combined with convective boundary condition via non-linearly stretched sheet. Najiyah et al. [30] discussed the impacts of velocity slip and convective boundary condition on $3 \mathrm{D}$ hybrid nanofluid flow past a stretching/shrinking surface. Rai et al. [31] analyzed the heterogeneous and homogeneous effects on the viscous fluid flow past a non-linear shaped stretchable surface with convective heating mechanism. Patel and Rajiv [32] studied the computational modelling of chemically radiative 
MHD mixed convection flows of microplar fluid past non-linear stretching surface embedded in porous media considering the effects of viscous dissipation, Joule heating and convective boundary condition. Sudarsana et al. [33] examined the heat flow behavior of nanofluid past a vertical cone embedded in porous media considering the effects of magnetic field, thermal radiation and chemical reaction of first order due to convective boundary condition. Gajjela and Garvandha [34] considered the $\mathrm{MHD}$ free convection flow, double-diffusion couple stress fluid past a stretching cylinder with surface imposed Robin boundary condition.

In thermodynamics, entropy is the sum of inaccessible energy in the process. Entropy refers to irreversibility of the device and the concept is widely seen in thermodynamics architecture. High entropy decreases the usable energy and has a significant effect on the efficiency of the device. Entropy demonstrates the means of separating the real materials through its components. In the irreversible processes, the initial state of the process and nearby environment cannot be preserved. Entropy and irreversibility analysis are directly proportional. Also, entropy tells us how the energy is utilized. The process is flexible due to the difference in temperature which induces energy transfer. The gradient of the system stimulates irreversibility to the process. Entropy leads to an increase in disorder in process which induces energy loss in the system and leads to a reduction in work potential. Thus, as we process the system, we restrict entropy generation and it is critical in various engineering applications, one of which is the power generation in power plants. Many researchers are focused on various approaches for entropy minimization in fluid flow problems. Bejan $[28,29]$ was the first to propose entropy generation minimization (EGM) to measure the disorders in isolated system. Rashad et al. [37] employed finite volume technique to investigate the entropy analysis and MHD mixed convection flow of Nanofluid in a lid driven U-shaped cavity with heat source. Mansour et al. [38] discussed the entropy generation and MHD mixed convection flow of Nanofluid in a C-shaped cavity with a corner heater and heat source. Chamkha et al. [39] presented the entropy generation analysis on MHD mixed convection flows of Nanofluid past a porous enclosure considering the effects of heat source/sink. Armaghani [40] studied the analytical simulation of thermal efficiency using entropy generation of MHD mixed convection flow of Nanofluid in an L-shaped duct using finite volume method. Fatunmbi and Adeniyan [41] reported the entropy generation and irreversibility analysis of nonlinearly radiative MHD micropolar fluid flow past a non-linear and non-permeable stretching surface using RK technique considering the effects of Joule heating and viscous dissipation. Li et al. [42] analyzed the entropy generation of 
radiative MHD nanofluid in a square cavity. Zafar et al. [43] presented the entropy generation of triple diffusive flow past a horizontal plate. Armaghani et al. [44] investigated the entropy generation in a porous I shaped cavity owing to MHD convection of Nanofluid in the presence of heat generation/absorption using finite difference technique.

There has been several literature reports in numerous perspectives relating to the dynamics of Tangent Hyperbolic liquid through an isothermal wedge. But as per the authors knowledge the non-similar solutions of Tangent Hyperbolic fluid past an isothermal wedge flows has not been considered. Therefore, the objective of the present study is to examine the MHD flow and heat transfer of tangent hyperbolic fluid through an isothermal wedge with convection boundary condition. Appropriate non-similar transformations are deployed to render the conservation equations dimensionless. The emerging non-dimensional partial differential equations with associated dimensionless boundary conditions constitute a highly nonlinear, coupled two-point boundary value problem making exact solutions practically impossible. The transformed dimensionless equations with appropriate boundary conditions are solved using the Keller's box implicit finite difference technique [45]. The impacts of various thermo-physical parameters, namely local Weissenberg mumber (We), local magnetic parameter (Ha), local biot mumber $(\gamma)$, mixed convection parameter ( $\lambda$ ), pressure gradient parameter $(m)$ and Prandtl mumber (Pr) on velocity, temperature, shear stress, entropy generation number, Bejan mumber and heat transfer rate are presented both graphically and numerically. The research is applicable for coating materials in chemical engineering for instance, robust paints, production of aerosol deposition and water soluble solution thermal treatment.

\section{TANGENT HYPERBOLIC FLUID MODEL}

Due to its simplicity, the current work employs a subclass of non-Newtonian fluids called the tangent hyperbolic fluid. The Cauchy stress tensor for a tangent hyperbolic fluid [10 - 11] is:

$$
\bar{\tau}=\left[\mu_{\infty}+\left(\mu_{0}+\mu_{\infty}\right) \tanh (\bar{\Gamma})^{n}\right] \bar{\gamma}
$$

where the shear rate, $y$ is defined as: 
$\dot{\gamma}=\sqrt{\frac{1}{2} \sum_{i j} \sum_{j} \gamma_{i j} \gamma_{j i}}=\sqrt{\frac{1}{2} \Pi}$

Here $\Pi=\frac{1}{2} \operatorname{tr}\left(\operatorname{gradV}+(\operatorname{gradV})^{T}\right)^{2}$. We consider the case where $\mu_{\infty}=0$ as numerical computations are not possible at infinite shear rate viscosity. Moreover, we confine to tangent hyperbolic fluid that describes shear thinning effects, i.e., $\Gamma \dot{\gamma}<1$. Taking these assumptions into consideration, Eqn. (1) can be written as,

$\left.\bar{\tau}=\mu_{0}\left(\begin{array}{c}\Gamma \dot{\gamma} \\ )^{n-} \dot{\gamma}=\mu_{0}[(1+\Gamma \dot{\gamma}-1\end{array}\right)^{n}\right]-\dot{\gamma}=\mu_{0}[1+n(\bar{\Gamma} \dot{\gamma}-1)]^{-}$

\section{MATHEMATICAL MODEL}

An incompressible, steady, laminar mixed convection flow of tangent hyperbolic fluid over an isothermal wedge with convective boundary condition and magnetohydrodynamic is examined. The physical schematic diagram of the problem is illustrated in Fig. 1. The $x$ (streamwise) and $y$ (transverse) coordinates are considered along the wedge and normal to the surface respectively. The gravitational acceleration, $g$ is considered to act downwards. A uniform magnetic field of strength $B_{0}$ is applied parallel to the $y$-axis i.e. transverse to the wedge surface. Magnetic induction, Hall current, ionslip and Maxwell displacement currents are neglected. Magnetic Reynolds number is small and the electric field due to polarization of charges is negligible. Both the fluid and wedge surface are initially held at a fixed temperature. Additionally, the electron pressure (for weakly conducting fluids) and the thermo-electric pressure are neglected. The applied magnetic field, $B_{0}$, is generated by passing a steady electric current along the longitudinal ( $z$-axis) parallel to the cone, where the wedge edges terminate at perfect electrodes which are connected via a load. Further, under usual boundary layer and Boussinesq approximations, the relevant equations for mass, linear momentum and energy are as follows:

$\frac{\partial u}{\partial x}+\frac{\partial v}{\partial y}=0$ 
$u \frac{\partial u}{\partial x}+v \frac{\partial u}{\partial y}=U_{\infty} \frac{d U_{\infty}}{d x}+v(1-n) \frac{\partial^{2} u}{\partial y^{2}}+\sqrt{2} v n \Gamma\left(\frac{\partial u}{\partial y}\right) \frac{\partial^{2} u}{\partial y^{2}} \pm g \beta \sin \left(\frac{\Omega}{2}\right)\left(T-T_{\infty}\right)-\frac{\sigma B_{0}^{2}}{\rho} u$

$u \frac{\partial T}{\partial x}+v \frac{\partial T}{\partial y}=\alpha \frac{\partial^{2} T}{\partial y^{2}}$

The non-Newtonian impacts only display in the shear terms in eqn. (5) and not in the convection term, though they often make the fluid dynamics quite intensely non-linear relative to the Newtonian fluids.

At $y=0, \quad u=0, \quad v=0, \quad-k \frac{\partial T}{\partial y}=h_{w}\left(T_{w}-T\right)$

As $y \rightarrow \infty, \quad u \rightarrow U_{\infty}=C x^{m}, \quad T \rightarrow T_{\infty}$

Here $m=\frac{\beta_{1}}{2-\beta_{1}}$ and $\beta_{1}=\frac{\Omega}{\pi}$. The stream function $\Psi$ is defined as $u=\frac{\partial \psi}{\partial y}$ and $v=-\frac{\partial \psi}{\partial x}$.

Consequently, Eqn. (4) is satisfied. The following dimensionless quantities are adopted:

$\xi=\frac{\sigma x}{\rho U_{\infty}}, \quad \eta=\frac{y}{x} \operatorname{Re}_{x}^{1 / 2}, \quad \psi=\left(U_{\infty} x v\right)^{1 / 2} f, \quad \theta=\frac{T-T_{\infty}}{T_{w}-T_{\infty}}, \quad \operatorname{Re}_{x}=\frac{U_{\infty} x}{v}$

In the light of Eqn. (8), the governing equations (5) - (7) can be written as:

$(1-n) f^{\prime \prime \prime}+\left(\frac{1+m}{2}\right) f f^{\prime \prime}+n W_{e} f^{\prime \prime} f^{\prime \prime \prime}+m\left(1-\left(f^{\prime}\right)^{2}\right)-H a f^{\prime}+\lambda \theta \sin \left(\frac{\Omega}{2}\right)=\xi(1-m)\left(f^{\prime} \frac{\partial f^{\prime}}{\partial \xi}-f^{\prime \prime} \frac{\partial f}{\partial \xi}\right)$

$\frac{\theta^{\prime \prime}}{\mathrm{Pr}}+\left(\frac{1+m}{2}\right) f \theta^{\prime}=\xi(1-m)\left(f^{\prime} \frac{\partial \theta}{\partial \xi}-\theta^{\prime} \frac{\partial f}{\partial \xi}\right)$

The transformed dimensionless boundary conditions are:

At $\quad \eta=0, \quad f=0, \quad f^{\prime}=0, \quad \theta=1+\frac{\theta^{\prime}}{\gamma}$

As $\eta \rightarrow \infty, \quad f^{\prime} \rightarrow 0, \quad \theta \rightarrow 0$

Here

$\gamma=\frac{x h_{w} \operatorname{Re}_{x}^{1 / 2}}{k}, \operatorname{Pr}=\frac{v}{\alpha}, \quad G r_{x}=\frac{g \beta_{1}\left(T_{w}-T_{\infty}\right) x^{3}}{v^{2}}, \quad W e=\frac{\sqrt{2} \Gamma \operatorname{Re}_{x}^{3 / 2}}{x^{2}}, \quad \lambda=\frac{G r_{x}}{\operatorname{Re}^{2}}$ and $H a=B_{0}^{2} x^{2} \frac{\sigma}{\mu}$

Due to non-similar nature of the flow, the parameters $W e, G r_{x}, y$ and $H a$ are local i.e., they are function of the tangential coordinate $x$. The thermal boundary condition at the wall in Eqn (12) 
refers to the convective cooling. The shear stress rate and heat transfer rate at the wedge surface are defined as:

$$
\begin{aligned}
\operatorname{Re}_{x}^{1 / 2} C_{f} & =(1-n) f^{\prime \prime}(\xi, 0)+\frac{n}{2} W_{c}\left[f^{\prime \prime}(\xi, 0)\right]^{2} \\
\operatorname{Re}_{x}^{1 / 2} N u & =-\theta^{\prime}(\xi, 0)
\end{aligned}
$$

\section{ENTROPY ANALYSIS}

The entropy analysis for the present flow regime in line with Bejan [35] is considered in this section. The volumetric rate of entropy generation due to magnetic field with heat transfer is given as:

$$
S_{g e n}^{*}=\frac{k}{T_{\infty}^{2}}\left(\frac{\partial T}{\partial y}\right)^{2}+\frac{\mu}{T_{\infty}}\left((1-n)+\frac{n \Gamma}{\sqrt{2}} \frac{\partial u}{\partial y}\right)\left(\frac{\partial u}{\partial y}\right)^{2}+\frac{\sigma B_{0}^{2} u^{2}}{T_{\infty}}
$$

The first term in eqn. (15) signifies the entropy generation produced by heat transfer, the second term denotes the entropy generation due to fluid friction and the third term is the entropy generation due to the magnetic Lorentz force. The non-dimensional entropy heat generation $(\mathrm{Ng})$ is defined as:

$$
N g=\frac{S_{g e n}^{-}}{S_{0}^{-}}=\left(\frac{\frac{k}{T_{\infty}^{2}}\left(\frac{\partial T}{\partial y}\right)^{2}+\frac{\mu}{T_{\infty}}\left((1-n)+\frac{n \Gamma}{\sqrt{2}} \frac{\partial u}{\partial y}\right)\left(\frac{\partial u}{\partial y}\right)^{2}+\frac{\sigma B_{0}^{2} u^{2}}{T_{\infty}}}{\frac{k(\Delta T)^{2}}{x^{2} T_{s}^{2}}}\right)
$$

$N g=\operatorname{Re}_{x} \theta^{12}+\frac{B r}{\Pi_{1}} \operatorname{Re}_{x}(1-n) f^{\prime 2}+\frac{n W e^{2}}{2} \frac{B r}{\Pi_{1}} \operatorname{Re}_{x} f^{\prime 3}+\frac{B r}{\Pi_{1}} H a f^{12}$

Here $B r=\frac{\mu u_{\infty}^{2}}{k \Delta T}, H a=B_{0}^{2} x^{2} \frac{\sigma}{\mu}, \Pi_{1}=\frac{\sigma}{\mu}$ and $\operatorname{Re}_{x}=\frac{U_{x} x}{v}$.

The Bejan mumber $(B e)$ is defined as the ratio of entropy heat generation due to heat and mass transfer to the overall entropy heat generation i.e.:

$$
B e=\frac{\text { Entropy due to Heat transfer }}{\text { Total entropy }}
$$




\section{NUMERICAL SOLUTION WITH IMPLICIT KELLER BOX METHOD (KBM)}

The non-dimensional Eqns. (10) - (12) form a $5^{\text {th }}$ order non-linear boundary value problem that needs a numerical solution. To solve this system of equations we implement the implicit finite differences scheme proposed by Keller known as Keller-Box Technique [45]. This approach remains highly popular and has retained comparative reliability than other numerical techniques. However, the drawback of this method is that because of its step, which must substitute the higher derivative with the first derivative, the computational effort per time step is costly, so that the second order derivatives can be written as a system of two first order derivatives. The discretized equations produce laborious algebraic expressions. The KBM however, has a second order accuracy with arbitrary spacing and appealing extrapolation characteristics. This method is very stable and outstanding precision is achieved. It converges easily and offers numerical meshing functions that are secure. The KBM offers an increase in the consistency of explicit or semiimplicit frameworks and uses a completely implicit methodology. For further details the readers can refer to [46] - [50]. The present KBM solutions are validated with the previous Newtonian computations. KBM comprises of the following 4 steps:

1. Reduction of Nth order partial differential system to $\mathrm{N}$ first order differential equations.

2. Finite difference discretization.

3. Newton quasilinearization of non-linear Keller algebraic equations.

4. Block-Tridiagonal elimination of Keller algebraic equations.

Step1: Reduction of the $N^{\text {th }}$ order partial differential equation system to $N$ first order equations New variables are introduced to Eqns. (10)-(12), to render the boundary value problem as a multiple system of first order equations. A set of five simultaneous first order differential equations are therefore generated by introducing the new variables:

$$
\begin{aligned}
& u(x, y)=f^{\prime}, v(x, y)=f^{\prime \prime}, s(x, y)=\theta, t(x, y)=\theta^{\prime} \\
& f^{\prime}=u \\
& u^{\prime}=v \\
& s^{\prime}=t \\
& (1-n) v^{\prime}+\frac{1+m}{2} f v+n W e v v^{\prime}+\left(1-u^{2}\right) m+\lambda \sin (\Omega) s-H a u=\xi(1-m)\left[u \frac{\partial u}{\partial \xi}-v \frac{\partial f}{\partial \xi}\right]
\end{aligned}
$$




$$
\frac{t^{\prime}}{\operatorname{Pr}}+\frac{1+m}{2} f=\xi(1-m)\left(u \frac{\partial s}{\partial \xi}-t \frac{\partial f}{\partial \xi}\right)
$$

where primes denote differentiation with respect to $\eta$. In terms of the dependent variables, the boundary conditions become:

$$
\begin{aligned}
& \text { At } \eta=0, \quad f=0, \quad u=0, \quad s=1 \\
& \text { As } \quad \eta \rightarrow \infty, \quad u \rightarrow 0, \quad s \rightarrow 0
\end{aligned}
$$

\section{Step 2: Finite Difference Discretization}

A two-dimensional computational grid is imposed on the $\xi \eta$ plane as sketched in Fig. 2. The stepping process is defined by:

$\eta_{0}=0, \quad \eta_{j}=\eta_{j-4}+h_{j}, \quad j=1,2, \ldots, J, \quad \eta_{J} \equiv \eta_{\infty}$

$\xi^{0}=0, \quad \xi^{n}=\xi^{n-1}+k_{n}, \quad n=1,2, \ldots, N$

where $k_{n}$ is the $\Delta \xi$ - spacing and $h_{j}$ is the $\Delta \eta$ - spacing. If $g_{j}^{n}$ denotes the value of any variable at $\left(\eta_{j}, \xi^{n}\right)$, then the variables and derivatives of Equations $(20)-(24)$ at $\left(\eta_{j-1 / 2}, \xi^{n-1 / 2}\right)$ are replaced by:

$$
\begin{aligned}
& g_{j-1 / 2}^{n-1 / 2}=\frac{1}{4}\left(g_{j}^{n}+g_{j-1}^{n}+g_{j}^{n-1}+g_{j-1}^{n-1}\right) \\
& \left(\frac{\partial g}{\partial \eta}\right)_{j-1 / 2}^{n-1 / 2}=\frac{1}{2 h_{j}}\left(g_{j}^{n}-g_{j-1}^{n}+g_{j}^{n-1}-g_{j-1}^{n-1}\right) \\
& \left(\frac{\partial g}{\partial g}\right)_{j-1 / 2}^{n-1 / 2}=\frac{1}{2 k^{n}}\left(g_{j}^{n}-g_{j-1}^{n}+g_{j}^{n-1}-g_{j-1}^{n-1}\right)
\end{aligned}
$$

The resulting finite - difference approximation of equations (20) - (24) for the mid - point $\left(\eta_{j-1 / 2}, \xi^{n}\right)$, are:

$$
\begin{aligned}
& h_{j}^{-1}\left(f_{j}^{n}-f_{j-1}^{n}\right)=u_{j-1 / 2}^{n} \\
& h_{j}^{-1}\left(u_{j}^{n}-u_{j-1}^{n}\right)=v_{j-1 / 2}^{n} \\
& h_{j}^{-1}\left(s_{j}^{n}-s_{j-1}^{n}\right)=t_{j-1 / 2}^{n}
\end{aligned}
$$




$$
\begin{aligned}
& (1-n)\left(v_{j}-v_{j-1}\right)+\frac{h_{j}}{4}\left(\frac{1+m}{2}+\alpha(1-m)\right)\left(f_{j}+f_{j-1}\right)\left(v_{j}+v_{j-1}\right)+\frac{n W e}{2}\left(v_{j}+v_{j-1}\right)\left(v_{j}-v_{j-1}\right) \\
& -(m-\alpha(1-m)) \frac{h_{j}}{4}\left(u_{j}+u_{j-1}\right)^{2}+m h_{j}+\frac{h_{j}}{2} \lambda \sin (\Omega)\left(s_{j}+s_{j-1}\right)-H a \frac{h_{j}}{2}\left(u_{j}+u_{j-1}\right) \\
& -(1-m) \frac{\alpha h_{j}}{2} f_{j-1 / 2}^{n-1}\left(v_{j}+v_{j-1}\right)+(1-m) \frac{\alpha h_{j}}{2} v_{j-1 / 2}^{n-1}\left(f_{j}+f_{j-1}\right)=\left[R_{1}\right]_{j-1 / 2}^{n-1} \\
& \frac{1}{\operatorname{Pr}}\left(t_{j}-t_{j-1}\right)+\frac{h_{j}}{4}\left(\frac{1+m}{2}+\alpha(1-m)\right)\left(f_{j}+f_{j-1}\right)\left(t_{j}+t_{j-1}\right)-(1-m) \frac{\alpha h_{j}}{4}\left(u_{j}+u_{j-1}\right)\left(s_{j}+s_{j-1}\right) \\
& +\frac{\alpha h_{j}}{2}(1-m) s_{j-1 / 2}^{n-1}\left(u_{j}+u_{j-1}\right)-\frac{\alpha h_{j}}{2}(1-m) u_{j-1 / 2}^{n-1}\left(s_{j}+s_{j-1}\right)-\frac{\alpha h_{j}}{2}(1-m) f_{j-1 / 2}^{n-1}\left(t_{j}+t_{j-1}\right) \\
& +(1-m) \frac{\alpha h_{j}}{2} t_{j-1 / 2}^{n-1}\left(f_{j}+f_{j-1}\right)=\left[R_{2}\right]_{j-1 / 2}^{n-1}
\end{aligned}
$$

where we have used the abbreviations

$$
\begin{gathered}
{\left[R_{1}\right]_{j-1 / 2}^{n-1}=-h_{j}\left[\begin{array}{l}
(1-n)\left(v^{\prime}\right)_{j-1 / 2}^{n-1}+\left(\frac{1+m}{2}-\alpha(1-m)\right)(f v)_{j-1 / 2}^{n-1}+n W e\left(v v^{\prime}\right)_{j-1 / 2}^{n-1} \\
-(m-\alpha(1-m))\left(u_{j-1}^{n-1}\right)^{2}+\lambda \sin (\Omega) s_{j-1 / 2}^{n-1}-H a u_{j-1 / 2}^{n-1}
\end{array}\right]} \\
{\left[R_{2}\right]_{j-1 / 2}^{n-1}=-h_{j}\left[\frac{1}{\operatorname{Pr}^{n}}\left(t^{\prime}\right)_{j-1 / 2}^{n-1}+\left(\frac{1+m}{2}-\alpha(1-m)\right)(f t)_{j-1 / 2}^{n-1}+\alpha(1-m)(u s)_{j-1 / 2}^{n-1}\right]}
\end{gathered}
$$

The boundary conditions are:

$f_{0}^{n}=u_{0}^{n}=0, s_{0}^{n}=1, u_{J}^{n}=0, s_{J}^{n}=0$

Stage 3: Quasilinearization of Non-Linear Keller Algebraic Equations

If we assume $f_{j}^{n-1}, u_{j}^{n-1}, v_{j}^{n-1}, s_{j}^{n-1}, t_{j}^{n-1}$ to be known for $0 \leq j \leq J$, this leads to a system of $5 J+5$ equations for the solution of $8 J+8$ unknowns $f_{j}^{n}, u_{j}^{n}, v_{j}^{n}, s_{j}^{n}, t_{j}^{n}, j=0,1,2, \ldots, J$. This non-linear system of algebraic equations is linearized by means of Newton's method.

\section{Stage 4: Block-tridiagonal Elimination Solution of Linear Keller Algebraic Equations}

The linearized system is solved by the block-elimination method, since it possess a blocktridiagonal structure. The bock-tridiagonal structure generated consists of block matrices. The complete linearized system is formulated as a block matrix system, where each element in the 
coefficient matrix is a matrix itself, and this system is solved using the efficient Keller-box method. The numerical results are strongly influenced by the number of mesh points in both directions. After some trials in the $\eta$-direction (radial coordinate) a larger number of mesh points are selected whereas in the $\xi$-direction (tangential coordinate) significantly less mesh points are utilized. $\eta_{\max }$ has been set at 8.0 and this defines an adequately large value at which the prescribed boundary conditions are satisfied. $\xi_{\max }$ is set at 3.0 for this flow domain Mesh independence is achieved in the present computations. The numerical algorithm is executed in MATLAB on a PC. The method demonstrates excellent stability, convergence and consistency, as elaborated by Keller [45].

\section{RESULTS AND DISCUSSION}

Table 1 provides the shear stress rate $\left(C_{f}\right)$ values for various $m$ values. The existing findings are compared with Khan and Pop [51], Yacob et al. [52] and White [53] and are found to be in excellent correlation that supports the reliability of the current KBM code. Table 2 illustrates the impacts of $W e, n, m, \gamma, H a$ and $\lambda$ on $C_{f}$ and $N u$ along with a variation in $\xi$. An increase in We is seen to enhance $C_{f}$ whereas $\mathrm{Nu}$ is reduced. The parameter $W e$ defines the relative effect of viscous force to elastic force for flows. It is perhaps inversely or directly related to viscous force. Also, it characterizes the association between the fluid's stress relaxation time and the particular time of operation. In general, Weissenberg number arises from scaling the evolution of stress in rheological research and embedded within this are available alternatives for fluids with various shear or elongation rate and length scales. From the def., $W e\left(\frac{\sqrt{2} \Gamma \mathrm{Re}_{x}^{3 / 2}}{x^{2}}\right)$ is directly proportional to Time dependent material constant $(T)$, fluid elasticity and inversely proportional to $v^{1 / 2}$. Hence the Weissenberg number is lowered for higher viscosity and as the velocity is decreased, this helps to maximize skin friction. As We reaches 0 , the flow become Newtonian. It is further noted that with rising $n$ values reduces $C_{f}$ but enhance $N u$. A rise in power law index induces the stream to differ from Newtonian flows and reduces the velocity and hence the shear stress also reduces. Also, rising $m$ is found to enhance both $C_{f}$ and $N u$. A rise in $\gamma$ is noted to enhance both $C_{f}$ and $N u$. It has been observed that a rise in $\lambda$ enhance both $C_{f}$ and $N u$. There is a significant decline in $C_{f}$ and $\mathrm{Nu}$ with higher $\mathrm{Ha}$ values ( $\mathrm{Ha}$ represents relative influence of Lorentz's magnetic drag force to viscous hydrodynamic and buoyancy forces). A rise in magnetic drag force obviously prevents 
the movement of boundary layer and allowing a retardation and a reduction in the skin friction. Hence, the momentum boundary layer thickness will be improved. Also, the thermal improvement coupled with an increase in magnetic field leads the fluid temperature to exceed the wall temperature that counteracts the heat transfer from wall to fluid body. Hence the heat transfer rate is essentially reduced.

Figs. 3 - 6 depicts the influence $W_{\ell}$ on velocity $\left(f^{\prime}\right)$, temperature $(\theta)$, entropy $(\mathrm{Ng})$ and Bejan number $(B e)$ profiles. A reduction in velocity is observed near-wall region, with rising $W e$ (fig. 3). The parameter We characterizes the association between the fluid's stress relaxation time and the particular time of operation. Hence for greater We values, the relaxation time is increased and hence the velocity decreases. There seems to be a low viscosity effect with greater $W e$ values and hence the flow is less deformable. The momentum development is hampered by tougher fluids (low elasticity) and the boundary layer is thickened due to the inverse relation between $W e$ and viscosity. However, such impact is not retained in the far field regime where the momentum diffusion is promoted with greater fluid relaxation and a week acceleration with higher $W e$ is induced. It can be intimately related to the alteration in tensile stresses in the structure of the rheological boundary layer when shifting from the near wall regime to the free stream regime. The effects of $W e$ is temperature consistent, while the velocity is turned over. Evidently, the rheology of the fluid is more closely related to the diffusion of momentum than to thermal diffusion. The latter is affected indirectly, i.e. by the primary influence of fluid viscoelasticity, which is a secondary effect. In the boundary layer, there is naturally a rivalry between elastic and viscous forces, which depends, among other variables, on the proximity to the wedge surface. In context of the evolution of temperature this is not clear. The presumption is that a consistent and uniform temperature distribution during the coating process should be achieved, regardless of the degree of elasticity of the liquid (e.g. spray paint). Indeed, as $W_{\ell}$ increases, the temperature distributions increase slightly (fig. 4) and hence the thermal thickness of the boundary layer also increases. Also in figs. 5 and 6 , it is observed that $\mathrm{Ng}$ is increased slightly for greater We values whereas $\mathrm{Be}$ is lowered for greater We values. Physically, the relaxation time rises with larger We values implies that fluid particles resist more that results in loss in heat.

Figs. 7 - 10 illustrates the influence $n$ on velocity $\left(f^{\prime}\right)$, temperature $(\theta), \mathrm{Ng}$ and $\mathrm{Be}$ profiles. The fluid velocity (Fig. 6) is significantly increased with increasing $n$. A rise in $n$ values shortens the fluid's friction and hence the velocity is increased. For greater $n$ values, the fluid 
passes on to a viscous medium and leaves elastic properties. Hence the momentum diffusion is increased and hydromagnetic boundary layer thickness is reduced. Conversely, temperature, $\mathrm{Ng}$ and $B e$ are reduced with a rise in $n$ values. An increase in $n$ values, steadily decreases temperature as momentum diffusion rate exceeds thermal diffusion rate resulting in cooling the boundary layer regime.

Figs. $11-14$ presents the influence $m$ on velocity $\left(f^{\prime}\right)$, temperature $(\theta), \mathrm{Ng}$ and $\mathrm{Be}$ profiles. It is reported with increasing $m$ values, the velocity increased significantly while the temperature fell considerably. For increasing $m$ values a huge rise in $\mathrm{Ng}$ and $\mathrm{Be}$ is observed. For $m=0$ in eqn. (10) the Blasius flow past a flat plate is achieved. The momentum boundary layer thickness is lowered efficiently with greater pressure gradient and the thermal boundary layer thickness is also reduced. With $m=1$, we obtain the case of flow on an infinite plate in the vicinity of the stagnation point flow. Although these two instances of flow are of mathematical interest, they are not in functional polymer production. For $m \neq 0$, the generalized wedge case is more important.

Figs. $15-18$ depict the influence $\lambda$ on velocity $\left(f^{\prime}\right)$, temperature $(\theta), N g$ and Be profiles. With increasing $\lambda$ values, a small rise in velocity is noted. By def., $\lambda$ represents the ratio of buoyancy force to the viscous force. Greater $\lambda$ values lead to improved natural convection. The viscous effect is less with greater $\lambda$ values and hence velocity increases. Physically, greater $\lambda$ values leads to increase in the convective cooling phenomenon and hence the fluid velocity increase. On the other hand, the fluid temperature is slightly reduced with increasing $\lambda$ values which is due to the acceleration in the convective cooling phenomenon of the system Fig. 17 reveals that there is an improvement in the $\mathrm{Ng}$ in near vicinity to the wedge surface with an increase in $\lambda$ values, although there is a decline further from the wedge surface. Also for $\eta>2$, the entropy converges asymptotically to zero. With increasing $\lambda$ values, the Bejan number is significantly enhanced near the surface and reduced far from the surface. Nevertheless, the Bejan number profiles converse at an intermediate distance from the wedge surface, suggesting that there is no tangible effect of the mixed convection in this area.

Figs. 19-22 presents the influence $\gamma$ on velocity $\left(f^{\prime}\right)$, temperature $(\theta), \mathrm{Ng}$ and $\mathrm{Be}$ profiles. With rising $\gamma$ values, a very small change i.e. a small increase in velocity is observed. And with increasing $\gamma$ values, a significant rise in temperature, $\mathrm{Ng}$ and $\mathrm{Be}$ is observed. A rise in Boit number 
increases the buoyancy force and the fluid carries the heat energy with higher velocity. Therefore, as $\gamma$ increases, the velocity also increases. The fluid considered in the present study is incompressible with respect to pressure but not temperature. Therefore, the fluid density changes with temperature variation due to thermal expandability and the flow is influenced by the buoyancy force. Hence, as $\gamma$ rises the temperature also rises. The entropy and Bejan numbers are also enhanced with a rise in Biot number. The regime is often reffered to as thermally simple for $\gamma<1$ and there is exists more consistent temperature fields within the boundary layer and solid wedge surface. The thermal fields are predicted to be nonlinear inside the solid wedge surface for $\gamma>1$. The Biot number essentially relates the conduction resistance inside a solid body to the convection resistance external to the body for heat transfer. It can also be observed that, $\gamma>0.1$ refers to a thermally dense component, while a thermally thin component is indicated for $\gamma<0.1$. By def, $\gamma$ is inversely proportional to thermal conductivity, hence the thermal conductivity at the wedge surface will decrease as the biot number increases and this leads to a reduction in the heat transfer rate from the boundary layer to inside the wedge, exhibiting in an increase in temperature at the wedge surface and the maximum effect at the surface will be preserved in the fluid body.

Figs. $23-26$ depict the influence $\Omega$ on velocity $\left(f^{\prime}\right)$, temperature $(\theta), N g$ and $B e$ profiles. When the inclination angle increases, the velocity is seen to increase slightly while the temperature distribution is found to decrease slightly. Also, the entropy generation number is seen to enhance as the inclination angle rises while the Bejan number is slightly reduced with increasing inclination angle.

Figs. $27-30$ depict the influence $H a$ on velocity $\left(f^{\prime}\right)$, temperature $(\theta), N g$ and Be profiles. Fig. 27 illustrates that the velocity profiles are depleted by increasing magnetic effects. Increasing the $\mathrm{Ha}$ values results in an improvement in the Lorentzian magnetohydrodynamic drag force. This Lorentz force reduces the shear effect on the wedge surface and thus decreases fluid velocity rapidly. The strong applied magnetic field resists the momentum flow which causes decline in the flow and thus increases the momentum boundary layer thickness. Alternatively, with an increase in Ha values, the temperature magnitudes are augmented slightly thereby increasing the thermal boundary layer thickness. Fig. 29 demonstrates that with an increase in $\mathrm{Ha}$, a significant downfall in $\mathrm{Ng}$ is produced. The Bejan number is notably augmented with an increase in $\mathrm{Ha}$ values. 
Figs. 31 - 34 illustrates the influence $B r$ and $R e$ on $\mathrm{Ng}$ and $\mathrm{Be}$ profiles respectively. The variations of $\mathrm{Br}$ on $\mathrm{Ng}$ and $\mathrm{Be}$ are illustrated in Figs. 31 \& 32 respectively. It is obvious from fig. 31 that the $\mathrm{Ng}$ profiles are greatly increased with increasing $\mathrm{Br}$ and the impact is more pronounced near the wedge surface. Initially, the energy is produced due to the dragged Lorentz forces produced which increases the entropy of the fluid. The thermal conductivity of the fluid declines with the rising $B r$, so that a greater amount of heat is transmitted through the fluid. The parameter $\mathrm{Br}$ characterizes the heat generated by viscous dissipation. This results in an enhancement in the entropy generation. Conversely, the Bejan number is reduced with respect to $\mathrm{Br}$ which is due to the dominant influence of viscous irreversibilities compared with irreversibilities in heat transfer. Figs. $33 \& 34$ presents the distributions of $\mathrm{Ng}$ and $\mathrm{Be}$ for various values of $\mathrm{Re}$. Both $\mathrm{Ng}$ and $\mathrm{Be}$ are increased with a rise in $R e$ values. The inertial force dominates the flow with reduced viscous force and results in a significant contribution of heat transfer to the entropy. The Reynolds number and entropy generation are proportional to each other. The Bejan number is also seen to increase with increasing values of $R e$.

\section{CONCLUSIONS}

The entropy generation of mixed convection flows of tangent hyperbolic fluid past isothermal wedge is considered. The Keller's implicit finite difference numerical technique is used to solve the modified, non-dimensional governing equations, subject to practical boundary conditions effectively. Absolutely brilliant association with prior research testifying to validity of this code is illustrated. The second law thermodynamics analysis of the flow is conducted by adopting the Bejan's entropy generation minimization approach. The calculations showed that:

I. An increase in $W e$ is seen to reduce velocity, $\mathrm{Ng}$ and $\mathrm{Be}$ whereas it elevates temperature.

II. Increasing $n$ increases the velocity whereas depresses temperature, entropy generation number and Bejan number.

III. An increase in $\gamma$ is seen to increase velocity, temperature, entropy generation number and Bejan number.

IV. An increase in $m$ is seen to increase velocity, $N g$ and $B e$ but reduces the temperature.

\section{REFERENCES}


[1] C. Fetecau, A.A. Zafar, D. Vieru, J. Awrejcewicz, Hydromagnetic flow over a moving plate of second grade fluids with time fractional derivatives having non-singular kernel, Chaos, Solitons \& Fractals, 130, 1094542020.

[2] S. Geethan Kumar, S.V.K. Varma, S.S.K. Raju, C.S.K. Raju, P. Durga Prasad, K. Durga Prasad, Three-dimensional conducting flow of radiative and chemically reactive Jeffrey fluid through porous medium over a stretching sheet with Soret and heat source/sink effects, Results in Engineering, 2020, 100139. hittps://doi.org/10.1016/j.rineng.2020.100139

[3] S.A. Shehzad, F. Mabood, A. Rauf, Iskander Tlili, Forced convective Maxwell fluid flow through rotating disk under the thermophoretic particles motion, Int. Comm. Heat and Mass Trans., 116, 2020, 104693. hittps://doi.org/10.1016/i.icheatmasstransfer. 2020.104693

[4] Panchun Liang, Shaowei Wang, Moli Zhao, Numerical study of rotating electroosmotic flow of Oldroyd-B fluid in a microchannel with slip boundary condition, Chinese Journal of Physics, 65, 2020, 459-471. https://doi.org/10.1016/i.ciph.2020.02.025

[5] M. Ijaz Khan, Mehr Nigar, T. Hayat, A. Alsaedi, On the numerical simulation of stagnation point flow of non-Newtonian fluid (Carreau fluid) with Cattaneo-Christov heat flux, Computer Methods and Programs in Biomedicine, 187, 2020, 105221. https://doi.org/10.1016/j.cmpb.2019.105221

[6] Meenakumari Ramamoorthy, Lakshminarayana Pallavarapu, Radiation and Hall effects on a 3D flow of MHD Williamson fluid over a stretchable surface, Heat Transfer, 2020;1-17. DOI: $10.1002 / \mathrm{htj} .21833$

[7] Sayer Obaid Alharbi, Abdullah Dawar, Zahir Shah, Waris Khan, Muhammad Idrees, Saeed Islam and I. Khan, Entropy Generation in MHD Eyring-Powell Fluid Flow over an Unsteady Oscillatory Porous Stretching Surface under the Impact of Thermal Radiation and Heat Source/Sink, Appl. Sci., 2018, 8, 2588; doi:10.3390/app8122588

[8] Mrutyunjay Das, Ganeswar Mahanta, Sachin Shaw, Heat and mass transfer effect on an unsteady MHD radiative chemically reactive Casson fluid over a stretching sheet in porous medium, Heat Transfer, 2020; 1-20. DOI: 10.1002/htj.21830

[9] Ali J. Chamkha, Mohamed Ahmed Mansour, Ahmed Mohamed Rashad, Hadi Kargarsharifabad and Taher Armaghani, Magnetohydrodynamic mixed convection and entropy analysis of Nanofluid in Gamma-Shaped porous cavity, Journal of Thermophysics and Heat Transfer, 2020. hittps://doi.org/10.2514/1.T5983 
[10] Zakir Ullah, Gul Zaman, Anuar Ishak, Magnetohydrodynamic tangent hyperbolic fluid flow past astretching sheet, Chinese J Phy., 66, 2020, 258-268.

[11] Wubshet Ibrahim, Tezera Gizewu, Nonlinear mixed convection flow of a tangent hyperbolic fluid with activation energy, Heat Transfer, 49(5), 2020, 2427-2448. https://doi.org/10.1002/htj.21729

[12] C. Kiparissides and J. Vlachopoulos, Finite element analysis of calendering, Polymer Engineering and Science, 16, $712-719$ (1976).

[13] I. S. Oyelakin, P. Sibanda, Analysis of exponentially varying viscosity and thermal conductivity on a tangent hyperbolic fluid, SeMA Joumal, 2020. https://doi.org/10.1007/s40324$\underline{020-00215-0}$

[14] M. Veera Krishna, Ali J. Chamkha, Hall and ion slip effects on MHD rotating boundary layer flow of nanofluid past an infinite vertical plate embedded in a porous medium, Results in Physics, 15, 102652. 2019. https://doi.org/10.1016/i.rinp.2019.102652

[15] B. Kumar, G.S. Seth, R. Nandkeolyar, Ali J. Chamkha, Outlining the impact of induced magnetic field and thermal radiation on magneto-convection flow of dissipative fluid, Int. J. Therm. Sci., 146, 106101, 2019. https://doi.org/10.1016/j.ijthermalsci.2019.106101

[16] Mamata Patil, Mahesha and C.S.K. Raju, Convective conditions and dissipation on Tangent hyperbolic fluid over a chemically heating exponentially porous sheet, Nonlinear Engineering, 8(1), 407-418, 2019. https://doi.org/10.1515/nleng-2018-0003

[17] S. Farooqa, M. Ijaz Khana, T. Hayat, M. Waqa, A. Alsaedib, Theoretical investigation of peristalsis transport in flow of hyperbolic tangent fluid with slip effects and chemical reaction, Journal of Molecular Liquids, 285, 2019, 314-322. https://doi.org/10.1016/j.molliq.2019.04.051 [18] K. Ganesh Kumar, B.J. Gireesha, R.S.R Gorla, Flow and heat transfer of dusty hyperbolic tangent fluid over a stretching sheet in the presence of thermal radiation and [19] Jitendra K Singh, Gauri Shanker Seth, S. Vishwanath, Pratima Rohidas, Steady MHD mixed convection flow of a viscoelastic fluid over a magnetized convectively heated vertical surface with Hall current and induced magnetic field effects, Heat Transfer, $2020 ; 1-24$. DOI: $10.1002 / \mathrm{htj} .21831$.

[20] K Venkatadri, S. Abdul Gaffar, P. Rajarajeswari, V. Ramachandra Prasad, O. Anwar Bég, B. Md. Hidayathulla Khan, Melting heat transfer analysis of electrically conducting nanofluid flow 
over an exponentially shrinking/stretching porous sheet with radiative heat fluxunder a magnetic field, Heat Transfer. 2020; 1-23. DOI: 10.1002/htj.21827.

[21] Samaira Aziz, Iftikhar Ahmad, Nasir Ali, Sami Ullah Khan, Magnetohydrodynamic mixed convection 3-D simulations for chemically reactive couple stress nanofluid over periodically moving surface with thermal radiation, Journal of Thermal Analysis and Calorimetry, 2020. https://doi.org/10.1007/s10973-020-09962-8

[22] L. Rosenhead, Laminar Boundary Layer's, Oxford, UK (1963).

[23] Chamkha, A.J., Abbasbandy, s., Rashad, A.M., Vajravelu, K., Radiation Effects of Mixed convection over a Wedge Embedded in a Porous Medium Filled with a Nanofluid, Transp. Porous Med., 91, 26-279, (2012) https://doi.org/10.1007/s11242-011-9843-5

[24] Takhar, H.S., Chamkha, A.J., Nath, G., Unsteady mixed convection flow from a rotating vertical cone with a magnetic field, Heat and Mass Trnasfer, 39, 297-304, 2003. httes://doi.org/101007/s00231-002-0400-1

[25] Gorla, R.S.R., Chamkha, A.J., Rashad, A.M., Mixed convective boundary layer flow over a vertical wedge embedded in a porous medium saturated with a Nanofluid: Natural convection dominated Regime, Nanoscale Res Lett, 6, 207, 2011. https://doi.org/10.1186/1556-276X-6-207 [26] Thameem Basha,H., Sivaraj, R., Subramanyam Reddy, A, Chamkha, A.J., SWCNH/diamondethylene glycol nanofluid flow over a wedge, plate and stagnation point with induced magnetic field and nonlinear radiation - solar energy application, Eur. Phys. J. Spec. Top., 228, 2531-2551, 2019. https://doi.org/10.1140/epjst/e2019-900048-x

[27] Chamkha, A.J., S. Abbasbandy, A.M. Rashad, K. Vajravelu, Radiation effects on mixed convection about a cone embedded in a porous medium filled with a Nanofluid, Meccanica, 48, 275-285, 2013. https://doi.org/10.1007/s11012-012-9599-1

[28] Nurul Amira Zainal, Roslinda Nazar, Kohilavani Naganthran, Ioan Pop, MHD mixed convection stagnation point flow of a hybrid nanofluid past a vertical flat plate with convective boundary condition, Chinese Journal of Physics, 66, 2020, 630-644. https://doi.org/10.1016/i.ciph.2020.03.022

[29] Tasawar Hayat, Rubina Riaz, Arsalan Aziz, Ahmed Alsaedi, Influence of Arrhenius activation energy in MHD flow of third grade nanofluid over a nonlinear stretching surface with convective heat and mass conditions, Physica A: Statistical Mechanics and its Applications, 549, 124006, 2020. https://doi.org/10.1016/j.physa.2019.124006 
[30] Najiyah Safwa Khashi' ie, Norihan Md Arifin, Ioan Pop, Roslinda Nazar, Ezad Hafidz Hafidzuddin, Nadihah Wahi, Three-Dimensional Hybrid Nanofluid Flow and Heat Transfer past a Permeable Stretching/Shrinking Sheet with Velocity Slip and Convective Condition, Chimese Journal of Physics, 66, 157-171, 2020. https://doi.org/10.1016/i.ciph.2020.03.032

[31] Rai Sajjad Saif, Taseer Muhammad, Haleema Sadia, Rahmat Ellahi, Boundary layer flow due to a nonlinear stretching curved surface with convective boundary condition and homogeneous and heterogeneous reactions, Physica A: Statistical Mechanics and its Applications, 551, 2020, 123996. https://doi.org/10.1016/j.physa.2019.123996

[32] Harshad R. Patela, Rajiv Singh, Thermophoresis, Brownian motion and non-linear thermal radiation effects on mixed convection MHD micropolar fluid flow due to nonlinear stretched sheet in porous medium with viscous dissipation, joule heating and convective boundary condition, Int. Comm. Heat and Mass Trans., 107, 2019, 68-92. hittps://doi.org/10.1016/i icheatmasstransfer.2019.05.007

[33] P. Sudarsana Reddy, P. Sreedevia, Ali J. Chamkha, Magnetohydrodynamic (MHD) boundary layer Q2 heat and mass transfer characteristics of nanofluid over a vertical cone under convective Q3 boundary condition, Propulsion and Power Research, 7 (4), 2018, 308-319. hittps://doi.org/10.1016/ijipor.2018.11.004

[34] N. Gajjela, M. Garvandha, The influence of magnetized couple stress heat, and mass transfer flow in a stretching cylinder with convective boundary condition, cross-diffusion, and chemical reaction, Thermal Science and Engineering Progress, 18, 2020, 100517. hittps://doi.org/10.1016/i.tsep.2020.100517

[35] A. Bejan, A study of entropy generation in fundamental convective heat transfer, $J$. Heat Transfer, 101 (1979) 718-725.

[36] A. Bejan, Second-law analysis in heat transfer and thermal design, Adv. Heat Transfer, 15 (1982) 1-58.

[37] A.M. Rashad, Mansour, M.A., T. Armaghani, A.J. Chamkha, MHD Mixed Convection and Entropy Generation of Nanofluid in a Lid-Driven U-Shaped Cavity with Internal Heat and Partial Slip, Physics of Fluids, 31, 042006 (2019). https://doi.org/10.1063/1.5079789

[38] Mansour, M.A., T. Armaghani, A.J. Chamkha, A.M. Rashad, Entropy Generation and Nanofluid Mixed Convection In A C-Shaped Cavity With Heat Corner and Inclined Magnetic 
Field, European Physical Journal Special Topics, 228(12) (2019) 2619-2645. httos://doi.org/10.1140/epist/e2019-900050-3

[39] A.J. Chamkha, A.M. Rashad, M.A. Mansour, T. Armaghani, M. Ghalambaz, Effects of heat sink and source and entropy generation on MHD mixed convection of a Cu-water Nanofluid in a lid-driven square porous enclosure with partial slip, Physics of Fluids, 29(5), 052001, 2017. https://doi.org/10.1063/1.4981911

[40] Armaghani, T., Rashad, A.M., Vahidifar, O., Mishra, S.R and Chamkha, A.J. (2019), Effects of discrete heat source location on heat transfer and entropy generation of nanofluid in an open inclined L-shaped cavity, International Journal of Numerical Methods for Heat \& Fluid Flow, Vol. 29 No. 4, pp. 1363-1377. https://doi.org/10.1108/HFF-07-2018-0412

[41] E.O. Fatunmbi, A. Adeniyan, Nonlinear thermal radiation and entropy generation on steady flow of magneto-micropolar fluid passing a stretchable sheet with variable properties, Results in Engineering, 6(2020) 1001422. https:/doi.org/10.1016/irineng 2020100142.

[42] Zhixiong Li, Ahmed Kadhim Hussein, Obai Younis, Masoud Afrand, Shizhe Feng, Natural convection and entropy generation of a nanofluid around a circular baffle inside an inclined square cavity under thermal radiation and magnetic field effects, International Communications in Heat and Mass Transfer, 116, 104620, 2020. https://doi.org/10.1016/i.icheatmasstransfer.2020.104650 [43] Zafar H. Khan, Waqar A. Khan, Jiguo Tang, Mikhail A. Sheremet, Entropy generation analysis of triple diffusive flow past a horizontal plate in porous medium, Chemical Engineering Science, 2020, 115980. https://doi.org/10.1016/j.ces.2020.115980

[44] Armaghani, T., Chamkha, A., Rashad, A.M., Inclined Magneto-Convection, Internal Heat and Entropy Generation of Nanofluid in an I-Shaped Cavity Saturated with Porous Media, J Therm Anal Calorim, 2020. https://doi.org/10.1007/s10973-020-09449-6

[45] Keller H.B., Numerical methods in boundary-layer theory, Ann. Rev. Fluid Mech. 10, 417433 (1978).

[46] H. Thameem Basha, R. Sivaraj, V. Ramachandra Prasad, O. Anwar Beg, Entropy generation of tangent hyperbolic nanofluid flow over a circular cylinder in the presence of nonlinear Boussinesq approximation: a non-similar solution, J Therm Anal Calorim, 2020. https://doi.org/10.1007/s10973-020-09981-5

[47] S. Abdul Gaffar, V. Ramachandra Prasad, O. Anwar Bég, Md. H. Hidayathullah Khan and K. Venkatadri, Radiative and magnetohydrodynamics flow of third grade viscoelastic fhid past an 
isothermal inverted cone in the presence of heat generation/absorption, J. Brazilian Soc. Mech Sci. Eng., 40:127-146(2018).

[48] K Madhavi, V. Ramachandra Prasad, S. Abdul Gaffar, Darcy flow of polymer from an inclined plane with convective heat transfer analysis: A numerical study, Journal of Thermal Analysis and Calorimetry, 2020. https://doi.org/10.1007/s10973-020-09942-y

[49] S. Abdul Gaffar, V. Ramachandra Prasad, E. Keshava Reddy, O. Anwar Beg, Magnetohydrodynamic free convection boundary layer flow of non-Newtonian Tangent Hyperbolic fluid from a vertical permeable cone with variable surface temperature, J of Brazilian Society of Mechanical Sciences and Engineering, 39, 101-116 (2017).

[50] B. Md. Hidayathulla Khan, S. Abdul Gaffar, O. Anwar Beg, Ali Kadir \& P. Ramesh Reddy, Computation of Eyring-Powell micropolar convective boundary layer flow from an inverted nonisothermal cone: thermal polymer coating simulation, Computational Thermal Sciences, 12(4):329-344 (2020).

[51] WA Khan, I Pop, Boundary hyer flow past a wedge moving in a nanofluid, Math Probl. Eng., 1-7 (2013).

[52] NA Yacob, A Ishak, I Pop, Falkner-Skan problem for a static or moving wedge in nanofluids.

Int. J. Therm Sci. 50, 2011 (2011).

[53] FM White, Viscous Fluid Flow, 2nd edn. (McGraw-Hill, New York, NY, USA, 1991.

\section{$\underline{\text { TABLES }}$}

Table 1: Comparison of the values of $C_{f}$ for various values of $m$ when $\lambda=0$

\begin{tabular}{|c|c|c|c|c|}
\hline$m$ & $\begin{array}{c}\text { Khan and } \\
\text { Pop [51] }\end{array}$ & $\begin{array}{c}\text { Yacob et } \\
\text { al. [52] }\end{array}$ & $\begin{array}{c}\text { White } \\
{[53]}\end{array}$ & $\begin{array}{c}\text { Present } \\
\text { results }\end{array}$ \\
\hline 0 & 0.4696 & 0.4696 & 0.4696 & 0.4696 \\
\hline $1 / 11$ & 0.6550 & 0.6550 & 0.6550 & 0.6549 \\
\hline $1 / 5$ & 0.8021 & 0.8021 & 0.8021 & 0.8019 \\
\hline $1 / 3$ & 0.9277 & 0.9276 & 0.9277 & 0.9275 \\
\hline $1 / 2$ & 1.0389 & 1.0388 & 1.0389 & 1.0387 \\
\hline 0 & 1.2326 & 1.2326 & 1.2326 & 1.2324 \\
\hline
\end{tabular}


Table 2: Values of $C_{f}$ and $N u$ for different $W_{\ell}, n, m, \gamma, \lambda, H a$ and $\xi$

$$
\left(\Omega=30^{\circ}, P r^{\prime}=7.0\right)
$$

\begin{tabular}{|c|c|c|c|c|c|c|c|c|c|c|c|}
\hline \multirow{2}{*}{$W_{e}$} & \multirow{2}{*}{$m$} & \multirow{2}{*}{$\lambda$} & \multirow{2}{*}{$\gamma$} & \multirow{2}{*}{$\mathrm{Ha}$} & \multicolumn{2}{|c|}{$\xi=1.0$} & \multicolumn{2}{|c|}{$\xi=2.0$} & \multicolumn{2}{c|}{$\xi=3.0$} \\
\hline & & & & & $C_{f}$ & $N u$ & $C_{f}$ & $N u$ & $C_{j}$ & $N u$ \\
\hline 0 & & & & & 0.4920 & 0.4431 & 0.4731 & 0.4357 & 0.4652 & 0.4327 \\
\hline 0.5 & & & & & & 0.5043 & 0.4386 & 0.4844 & 0.4315 & 0.4760 & 0.4286 \\
\hline 2 & & & & & & 0.5360 & 0.4284 & 0.5136 & 0.4215 & 0.5042 & 0.4188 \\
\hline 3 & & & & & & 0.5541 & 0.4230 & 0.5304 & 0.4163 & 0.5204 & 0.4136 \\
\hline 5 & & & & & & 0.5858 & 0.4144 & 0.5599 & 0.4079 & 0.5490 & 0.4053 \\
\hline & 0 & & & & & 0.5893 & 0.4228 & 0.5667 & 0.4156 & 0.5573 & 0.4127 \\
\hline & 0.25 & & & & & 0.5153 & 0.4370 & 0.4952 & 0.4298 & 0.4868 & 0.4269 \\
\hline & 0.5 & & & & & 0.4356 & 0.4560 & 0.4151 & 0.4486 & 0.4079 & 0.4457 \\
\hline & 0.75 & & & & & 0.3374 & 0.4827 & 0.3226 & 0.4753 & 0.3165 & 0.4725 \\
\hline & 0.85 & & & & & 0.2947 & 0.4967 & 0.2809 & 0.4895 & 0.2753 & 0.4868 \\
\hline & & 0.1 & & & & 0.2549 & 0.3197 & 0.2507 & 0.3191 & 0.2492 & 0.3191 \\
\hline & & 0.2 & & & & 0.3242 & 0.3550 & 0.3173 & 0.3536 & 0.3144 & 0.3528 \\
\hline & & 0.3 & & & & 0.3873 & 0.3870 & 0.3763 & 0.3832 & 0.3716 & 0.3814 \\
\hline & & 0.4 & & & & 0.4454 & 0.4152 & 0.4298 & 0.4092 & 0.4234 & 0.4068 \\
\hline & & 0.5 & & & & 0.4995 & 0.4404 & 0.4800 & 0.4331 & 0.4718 & 0.4302 \\
\hline & & & 0 & & & 0.4692 & 0.4357 & 0.4491 & 0.4277 & 0.4407 & 0.4246 \\
\hline & & & 2 & & & 0.5874 & 0.4540 & 0.5692 & 0.4481 & 0.5615 & 0.4458 \\
\hline & & & 4 & & & 0.6989 & 0.4708 & 0.6818 & 0.4660 & 0.6746 & 0.4641 \\
\hline & & & 6 & & & 0.8052 & 0.4860 & 0.7890 & 0.4819 & 0.7821 & 0.4803 \\
\hline & & & 9 & & & 0.9572 & 0.5062 & 0.9419 & 0.5029 & 0.9355 & 0.5016 \\
\hline & & & & 0.3 & & 0.4794 & 0.1458 & 0.4595 & 0.1432 & 0.4512 & 0.1422 \\
\hline & & & & 0.5 & & 0.4995 & 0.4404 & 0.4800 & 0.4331 & 0.4718 & 0.4302 \\
\hline & & & & 1 & & 0.5145 & 0.6641 & 0.4952 & 0.6536 & 0.4871 & 0.6493 \\
\hline & & & & 1.5 & & 0.5194 & 0.7391 & 0.5002 & 0.7276 & 0.4922 & 0.7230 \\
\hline & & & & 2 & & 0.5219 & 0.7768 & 0.5027 & 0.7647 & 0.4947 & 0.7599 \\
\hline & & & & & 0 & 0.5503 & 0.4577 & 0.5312 & 0.4165 & 0.5232 & 0.4485 \\
\hline & & & & & 0.05 & 0.5235 & 0.4489 & 0.5044 & 0.4246 & 0.4964 & 0.4392 \\
\hline & & & & & 0.1 & 0.4995 & 0.4404 & 0.4800 & 0.4331 & 0.4718 & 0.4302 \\
\hline & & & & & 0.15 & 0.4774 & 0.4323 & 0.4577 & 0.4419 & 0.4494 & 0.4216 \\
\hline & & & & & 0.2 & 0.4574 & 0.4245 & 0.4374 & 0.4511 & 0.4290 & 0.4133 \\
\hline
\end{tabular}




\section{FIGURES}

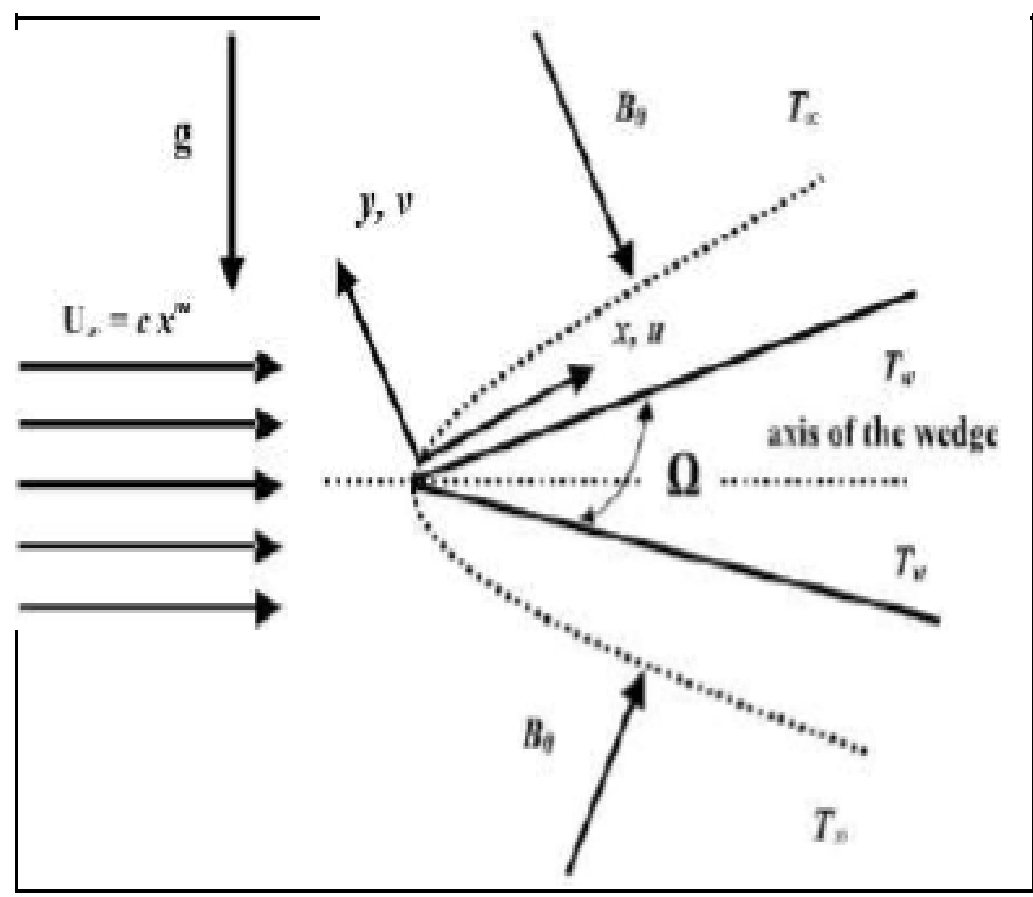

Fig. 1 Flow analysis along the wall of the edge

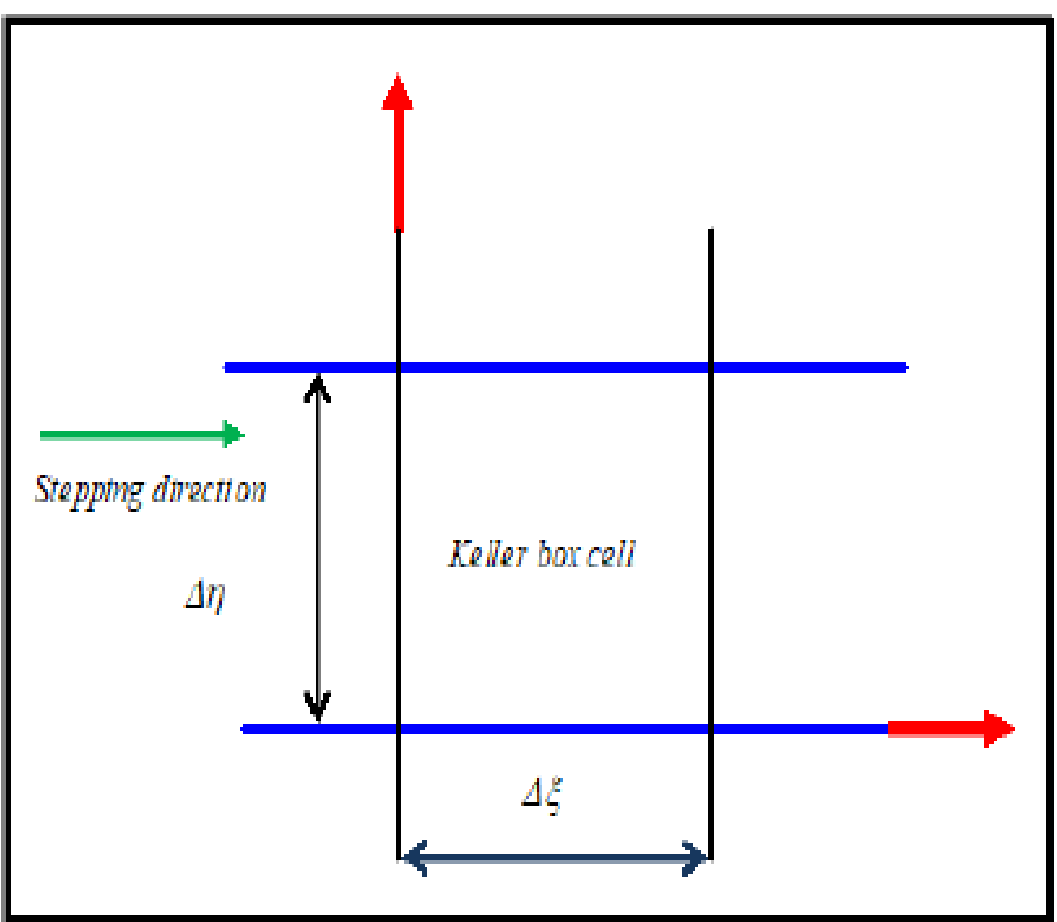

Fig. 2: Keller box computational cell 


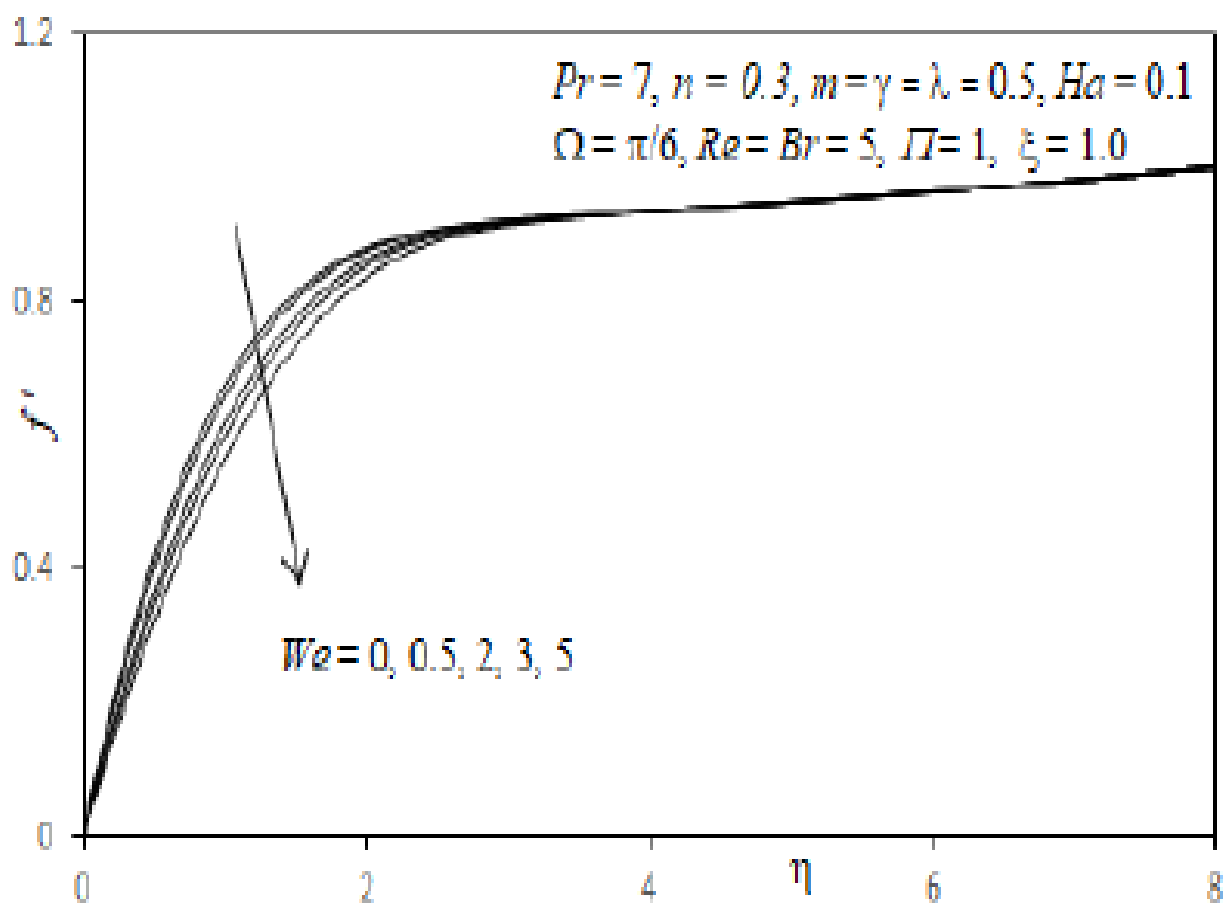

Fig. 3 Influence of We on Velocity Profiles

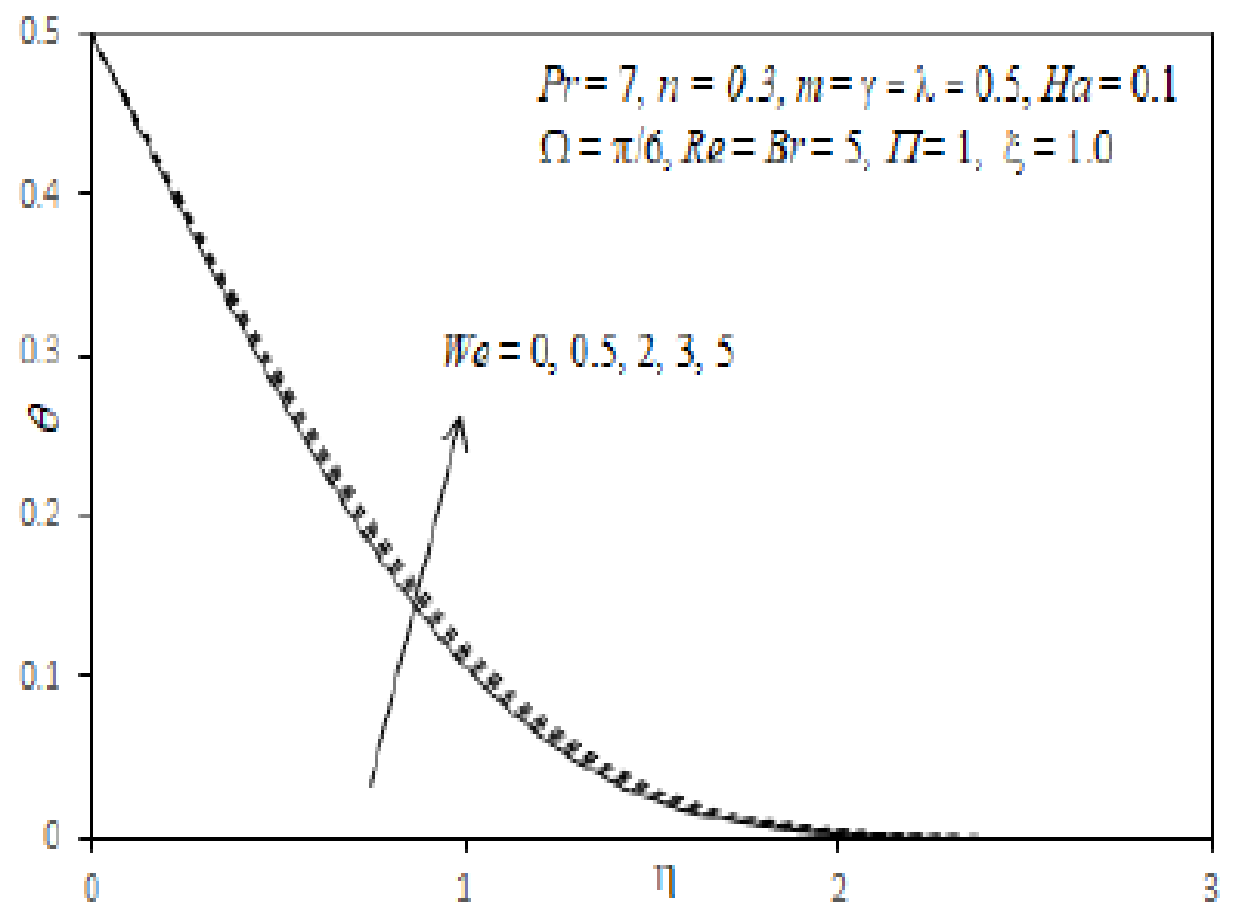

Fig. 4 Influence of We on Temperature Profiles 


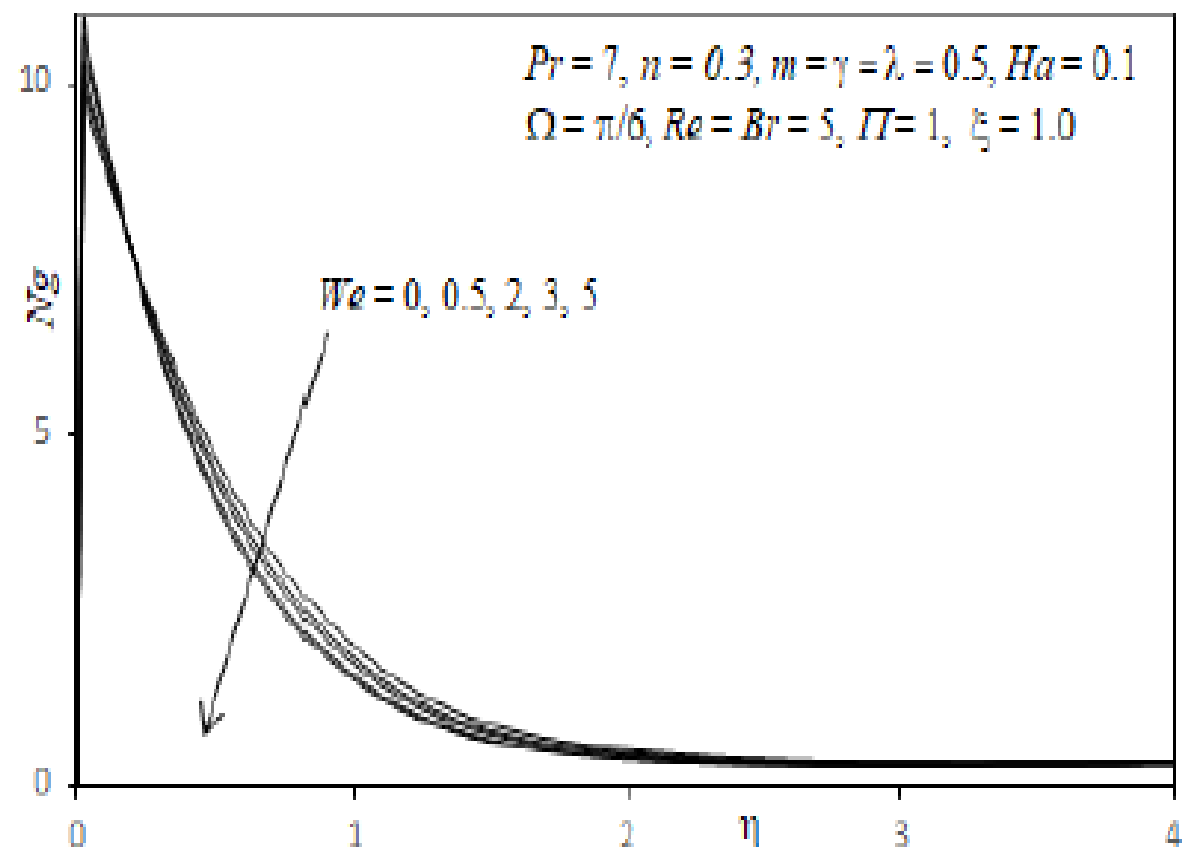

Fig. 5 Influence of Te on Entropy

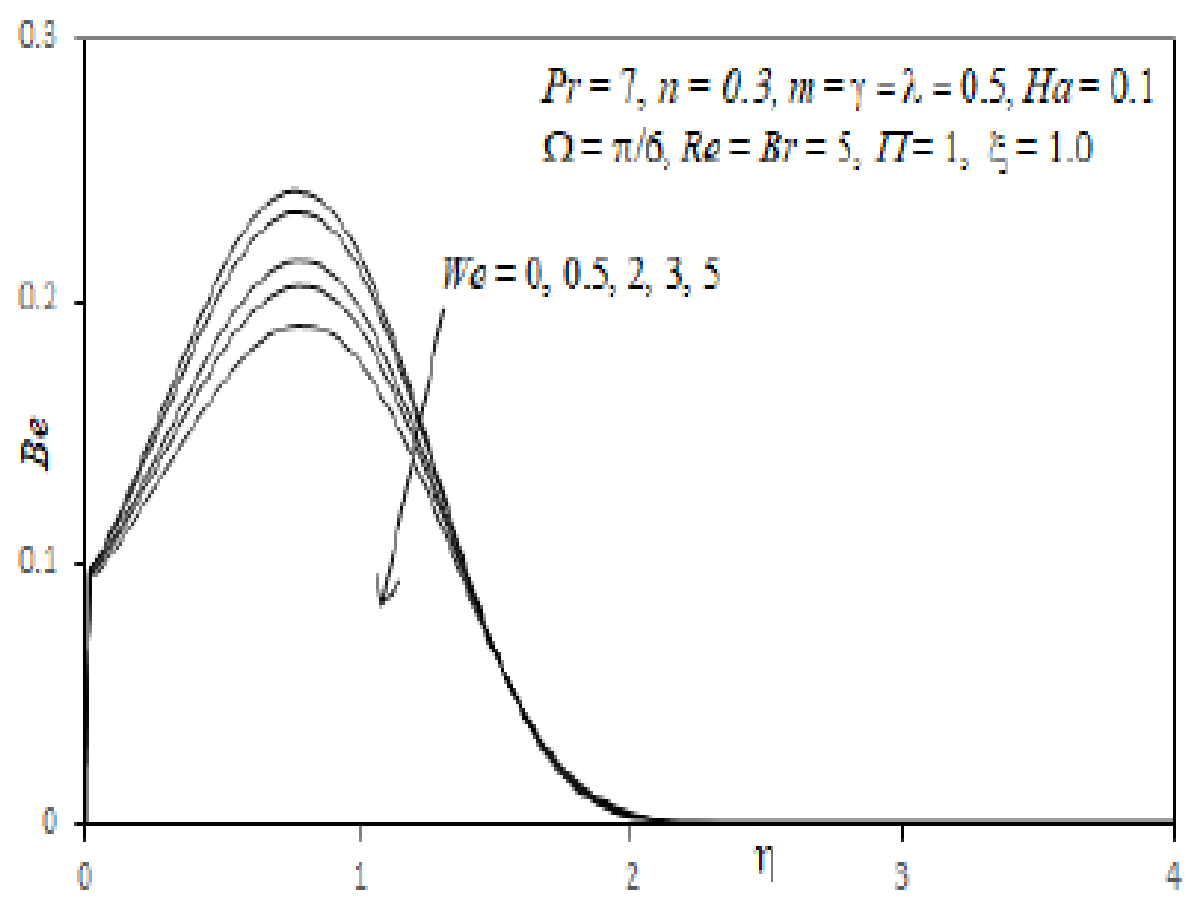

Fig. 6 Influence of We on Bejan Number 


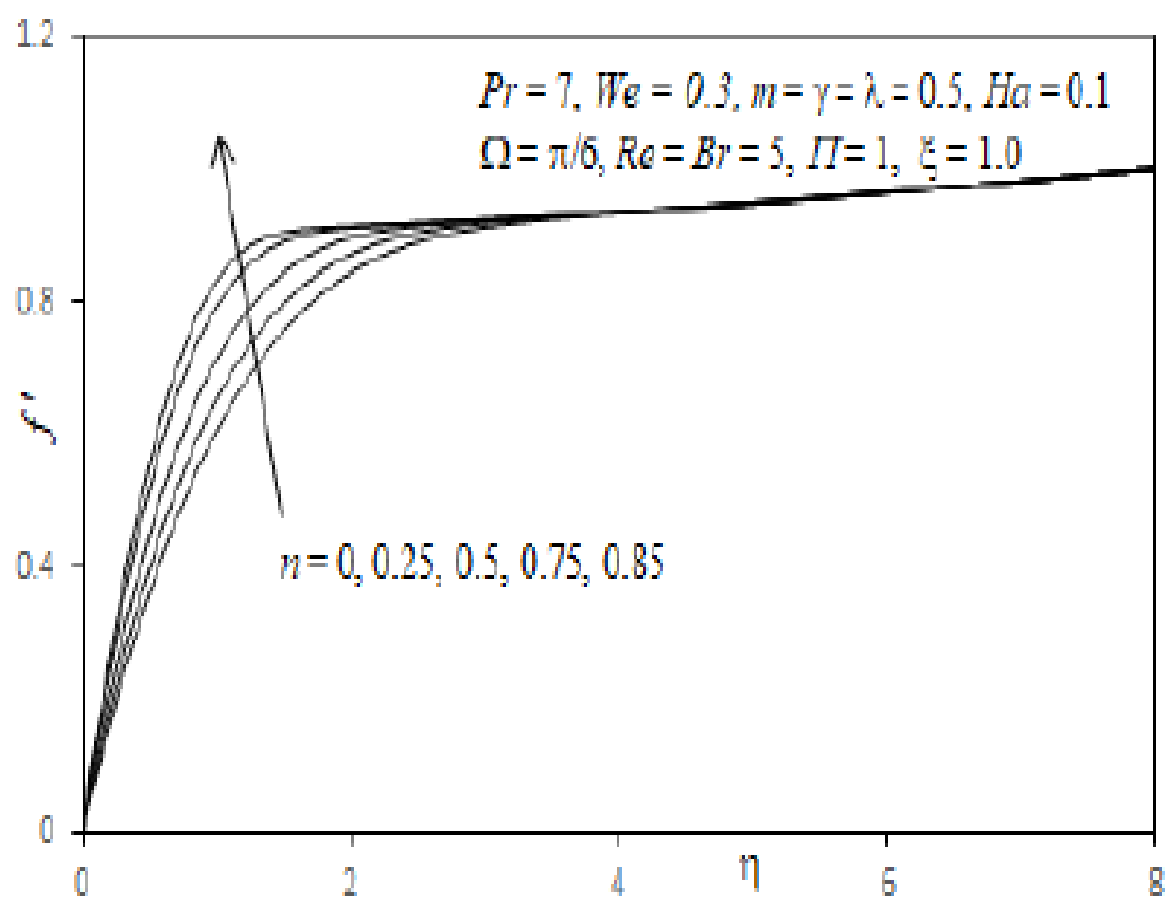

Fig. 7 Influence of $n$ on Velocity Profiles

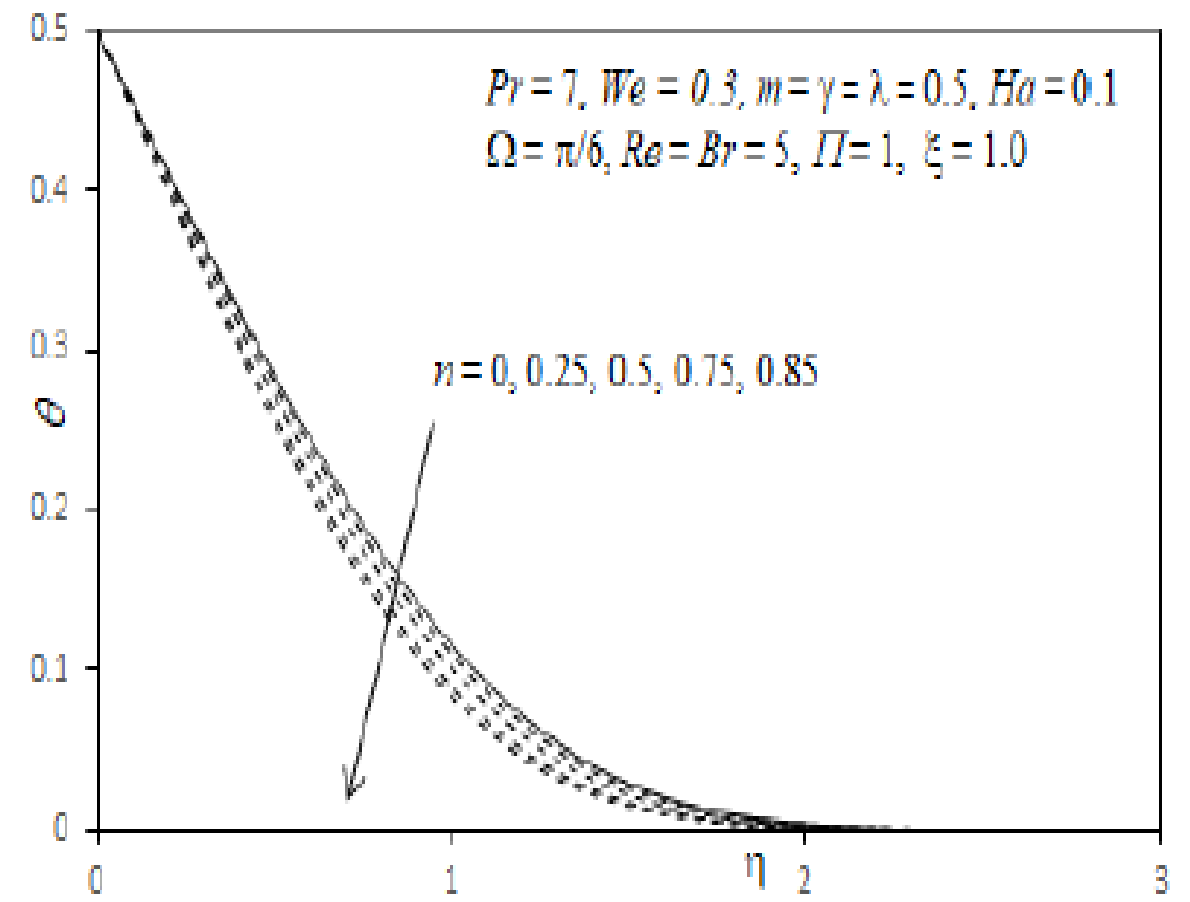

Fig. 8 Influence of $n$ on Temperature Profiles 


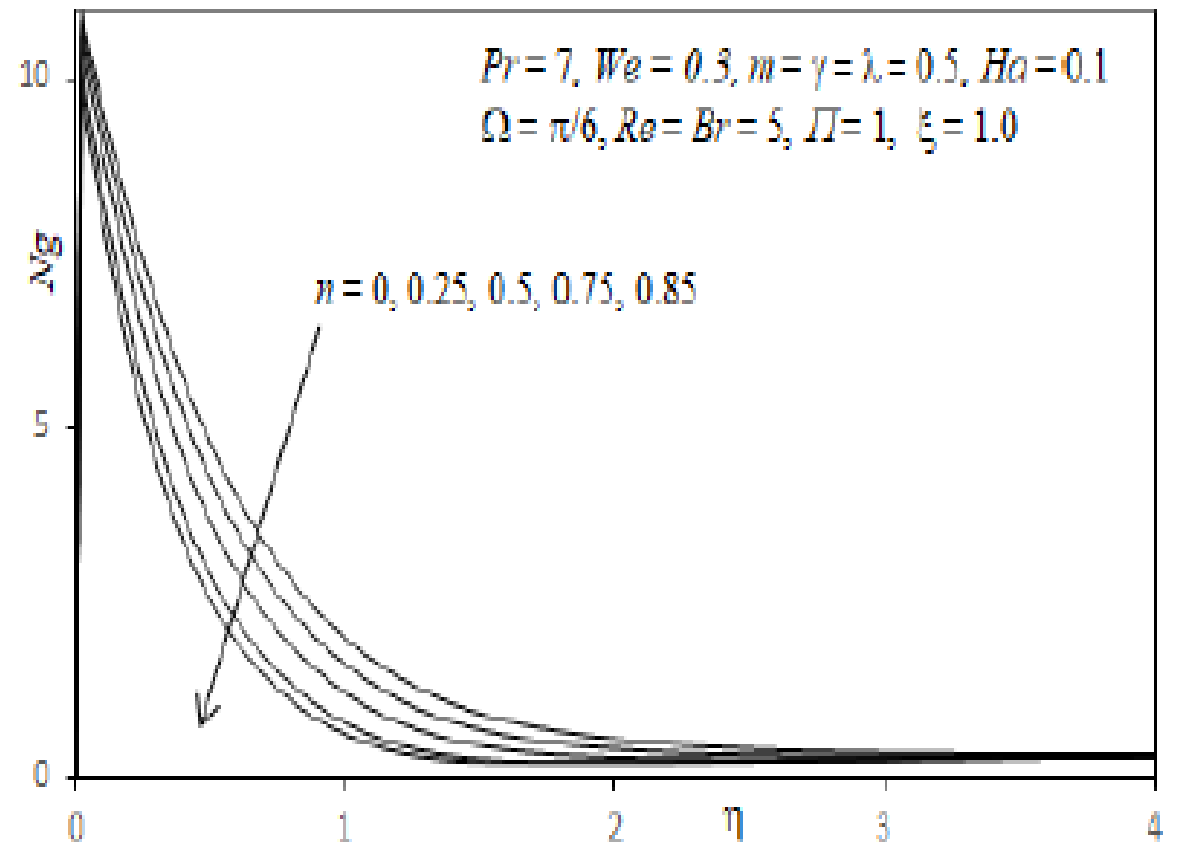

Fig. 9 Influence of $n$ on Entropy

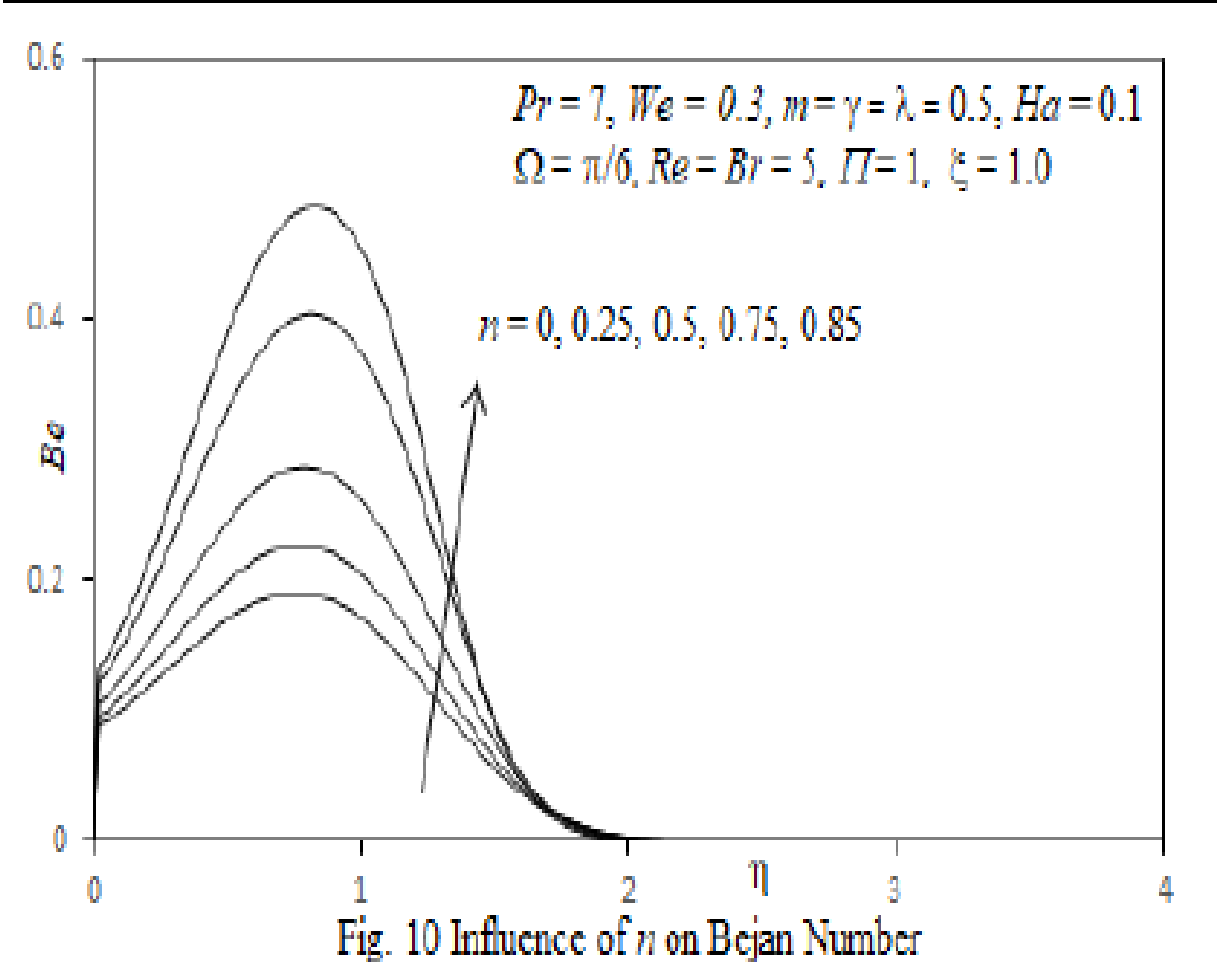




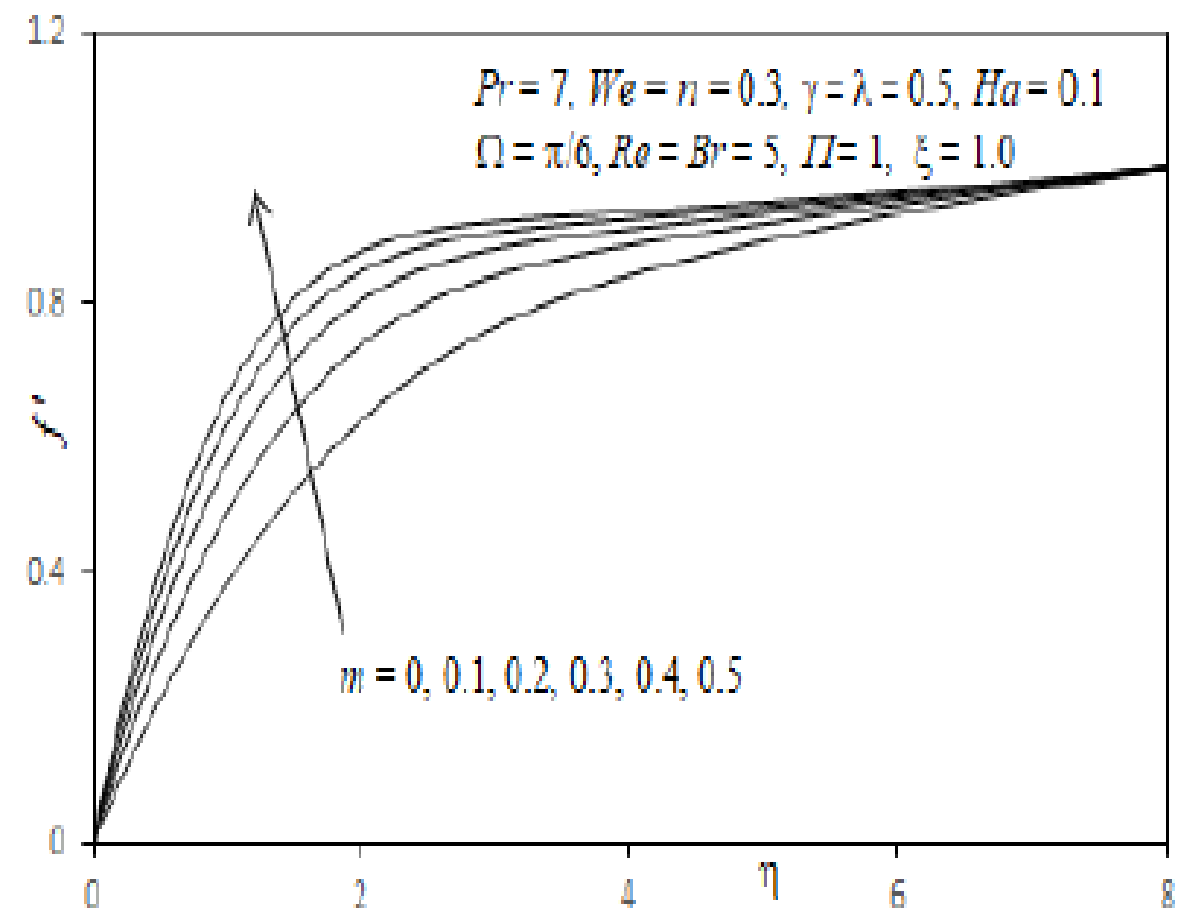

Fig. 11 Influence of $n$ on Velocity Profiles

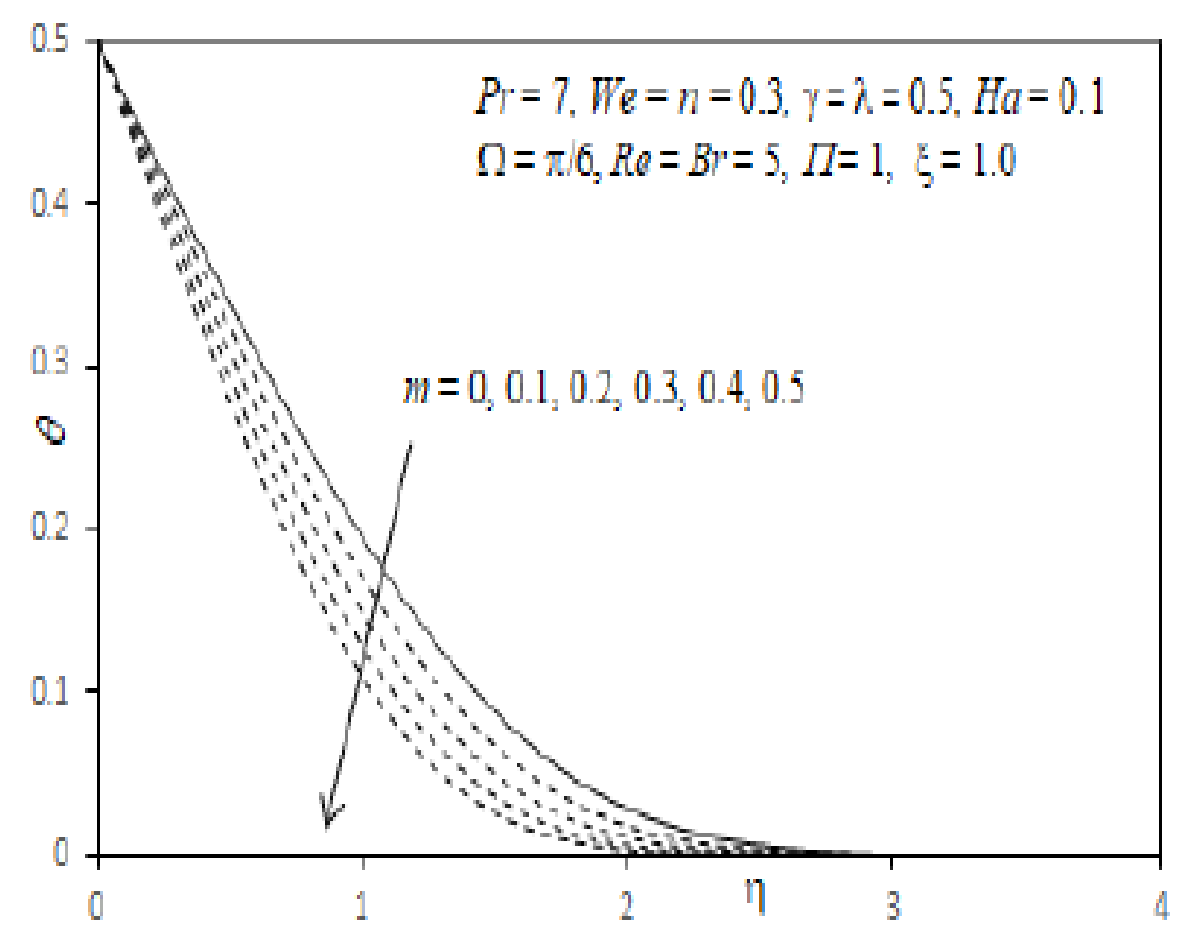

Fig. 12 Influence of $m$ on Temperature Profiles 


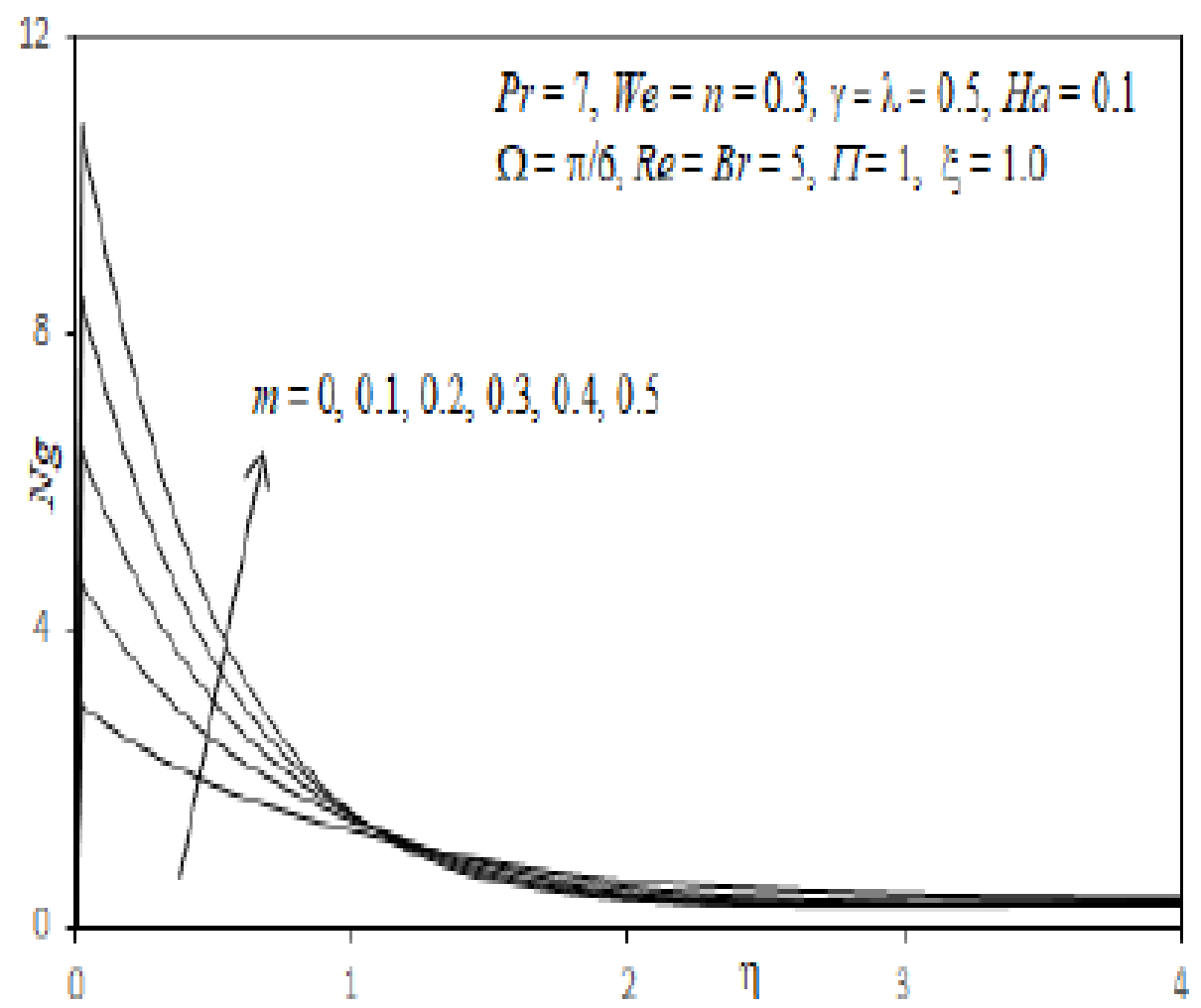

Figg. 13 Influence of in on Entropy

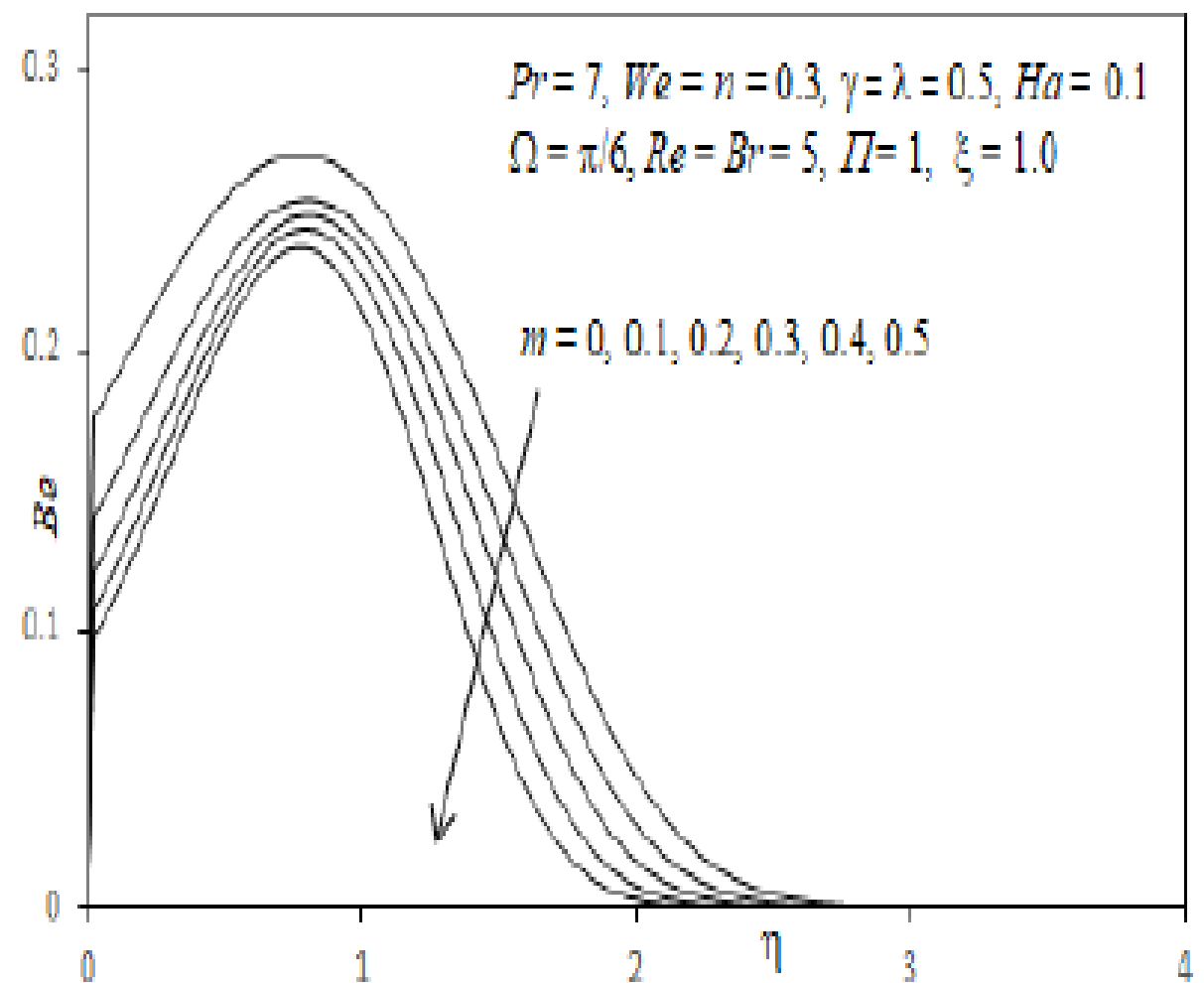

Fig. 14 Influence of $n$ on Bejan Number 


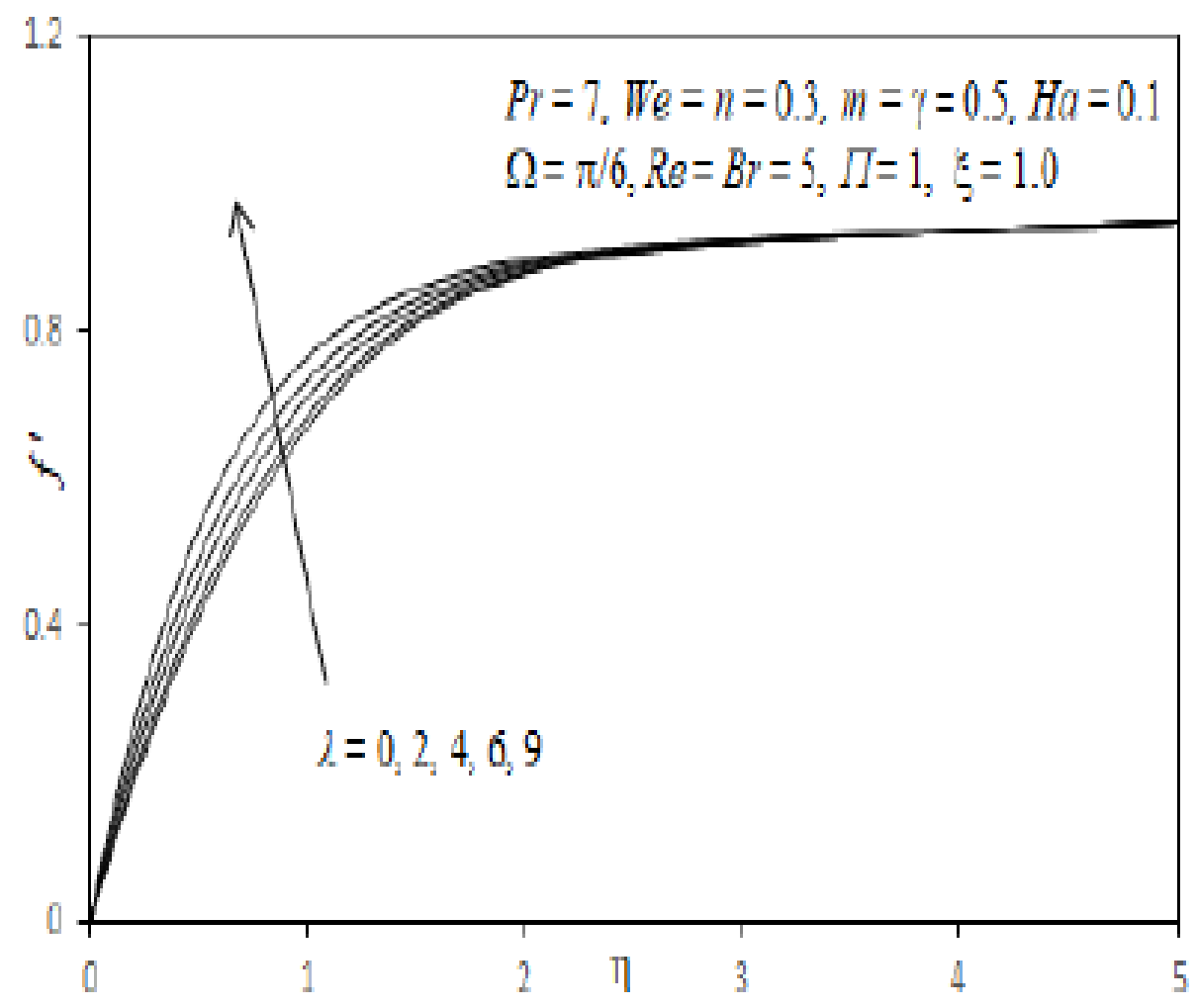

Fig. 15 Influence of $\lambda$ on Velocity Profiles

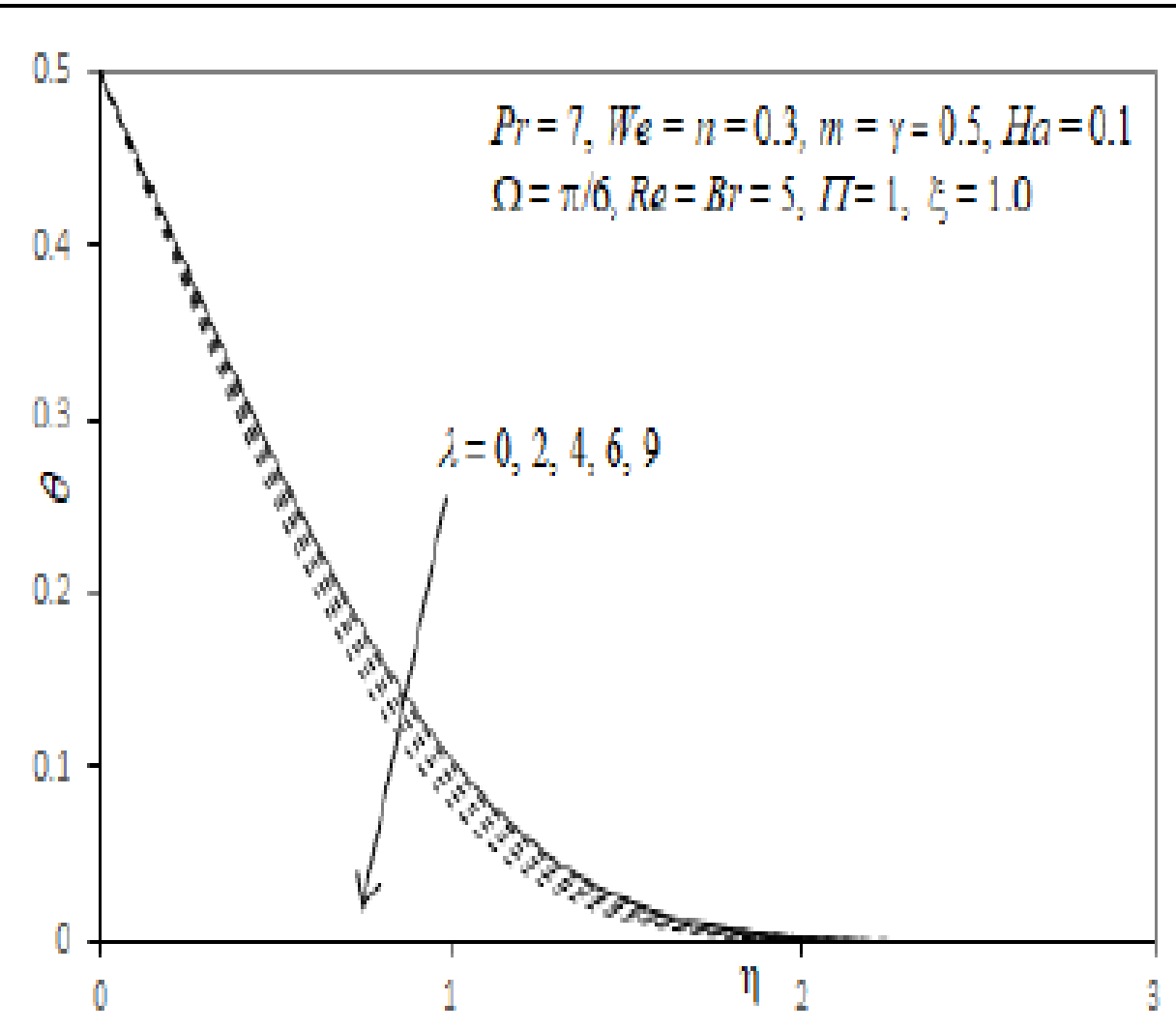

Fig. 16 Influence of $\lambda$ on Temperature Profles 


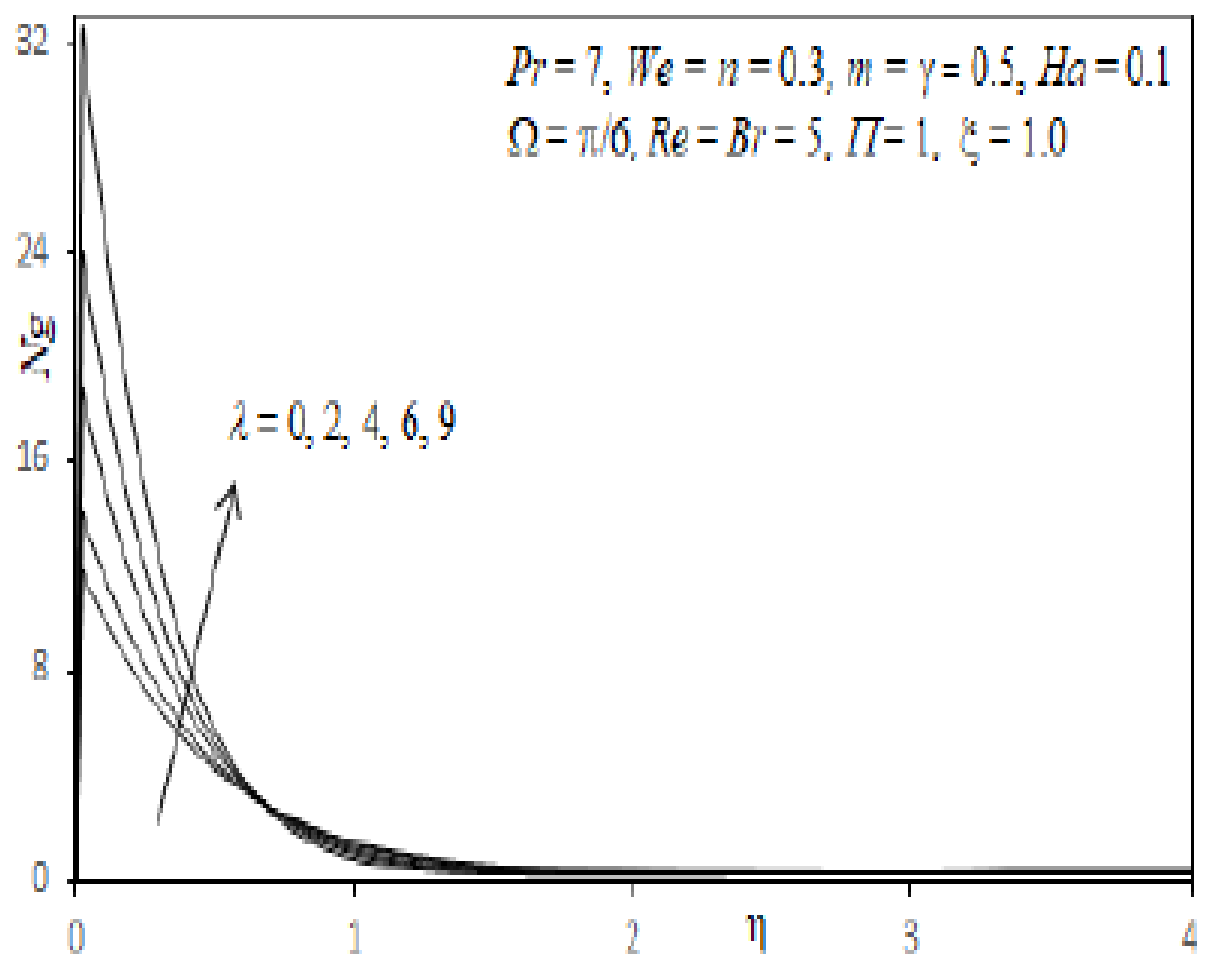

Fig. 17 Influence of $\lambda$ on Entropy

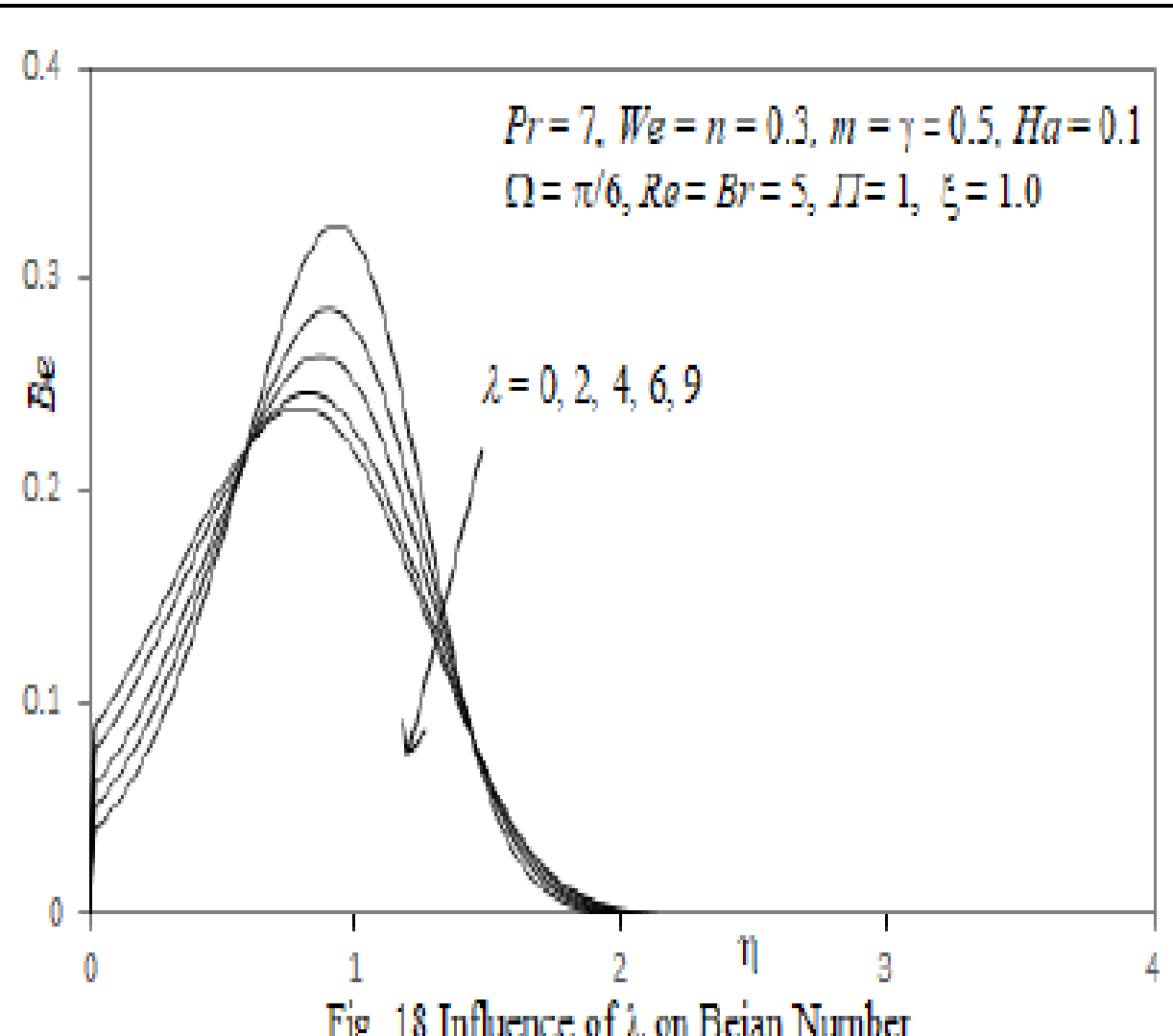

Fig. 18 Influence of $\lambda$ on Bejan Nunber 


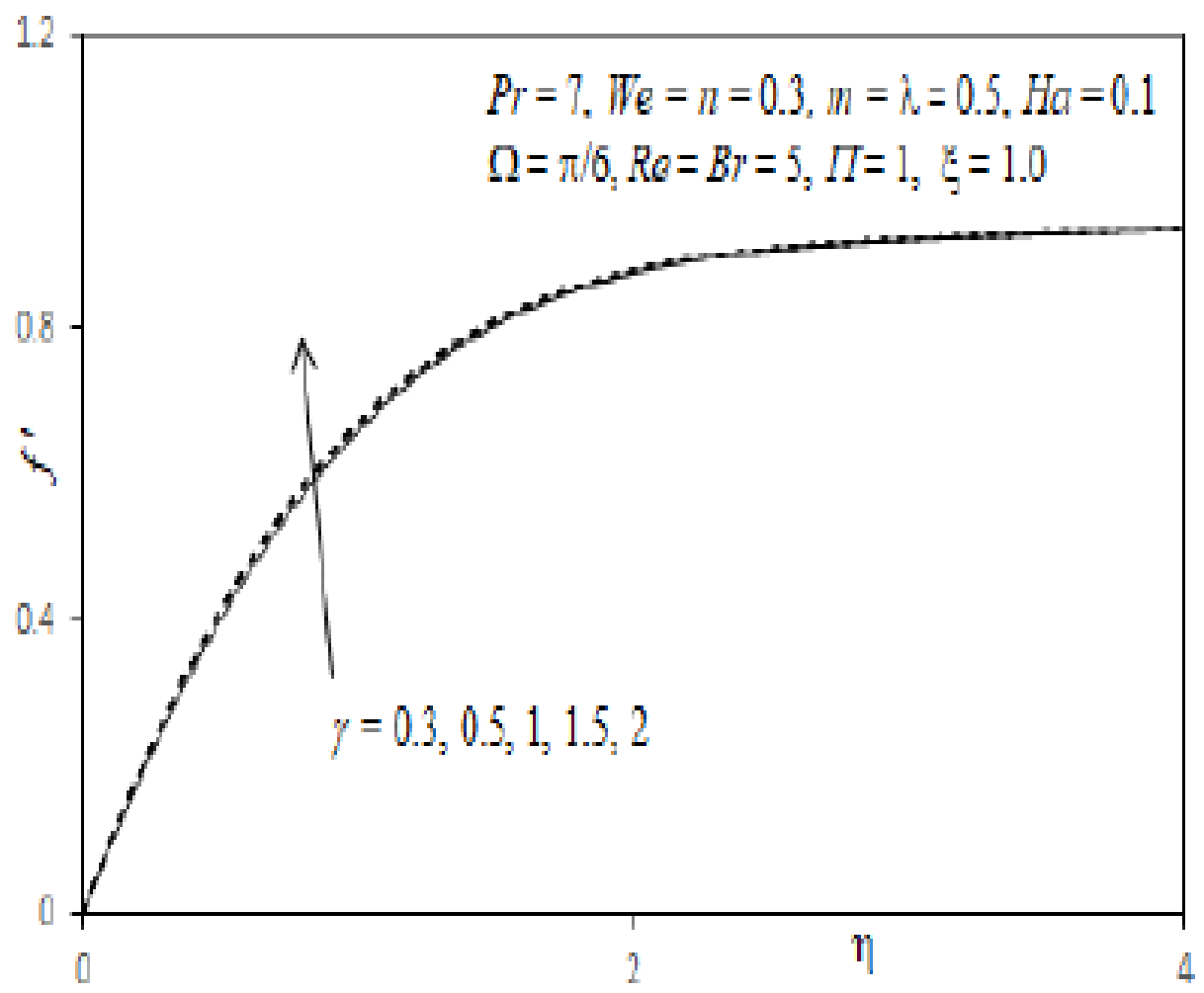

Fig. 19 Infuence of $\gamma_{\text {on }}$ Velocity Profiles

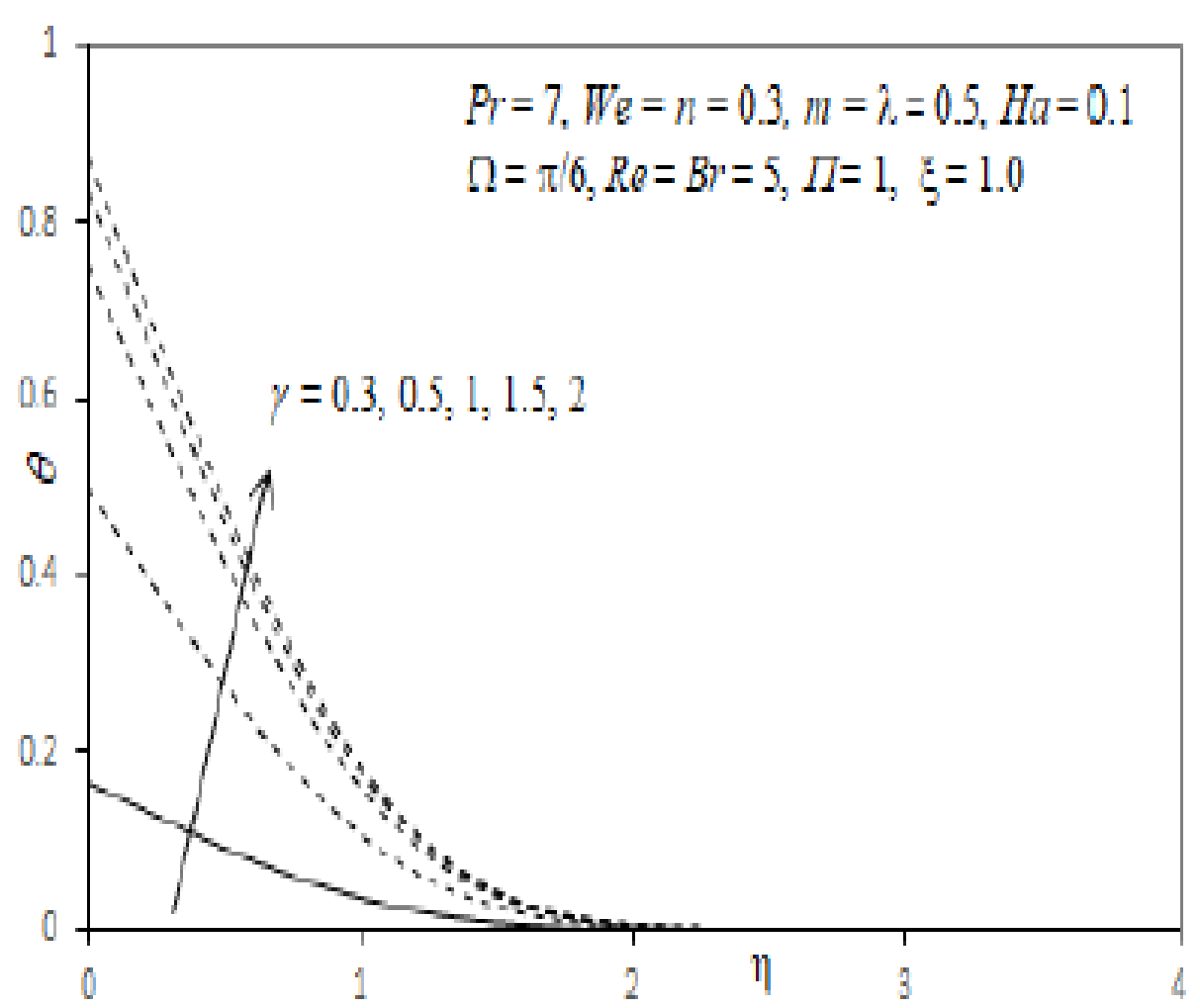

Fig. 20 Influence of $\gamma$ on Tempesature Profles 


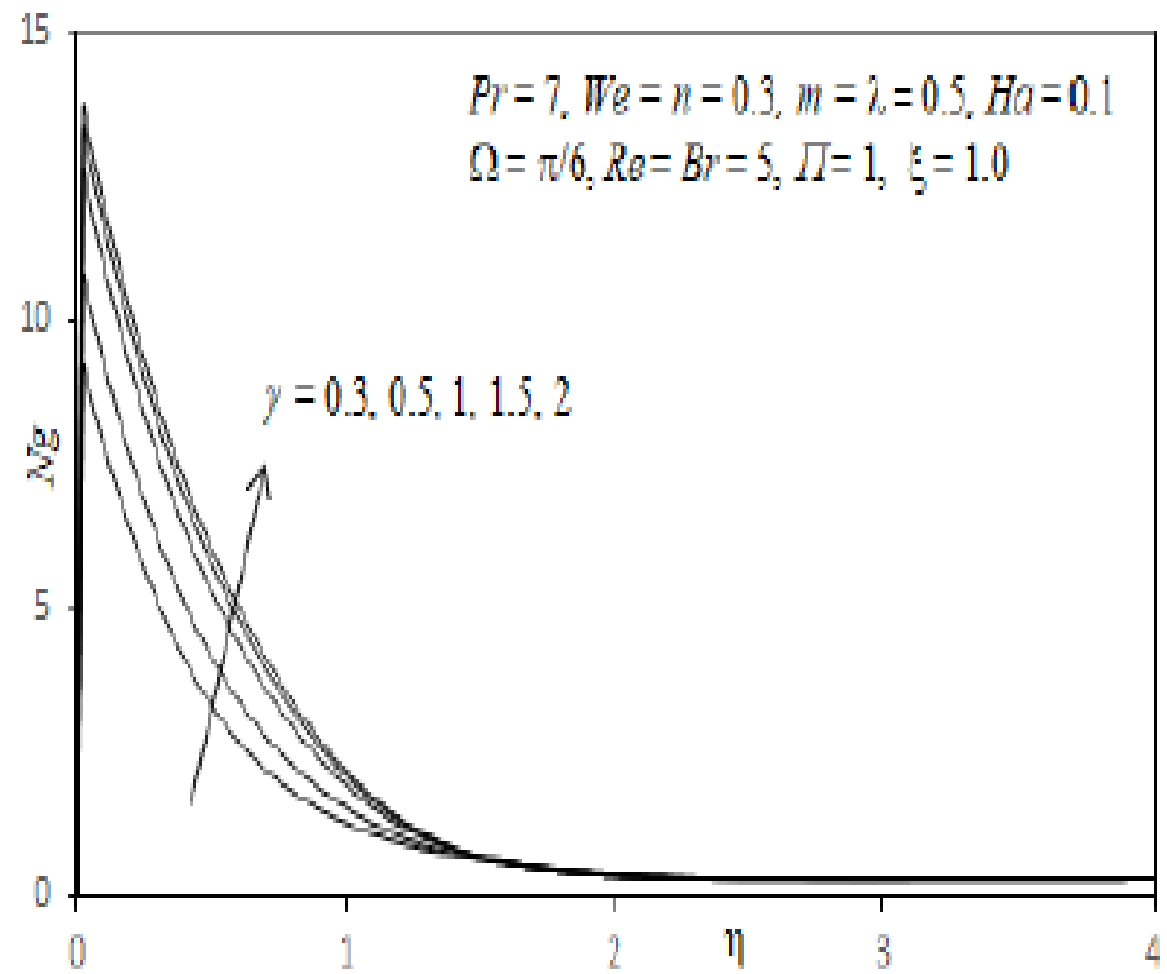

Fig. 21 Influence of $\gamma$ on Entropy

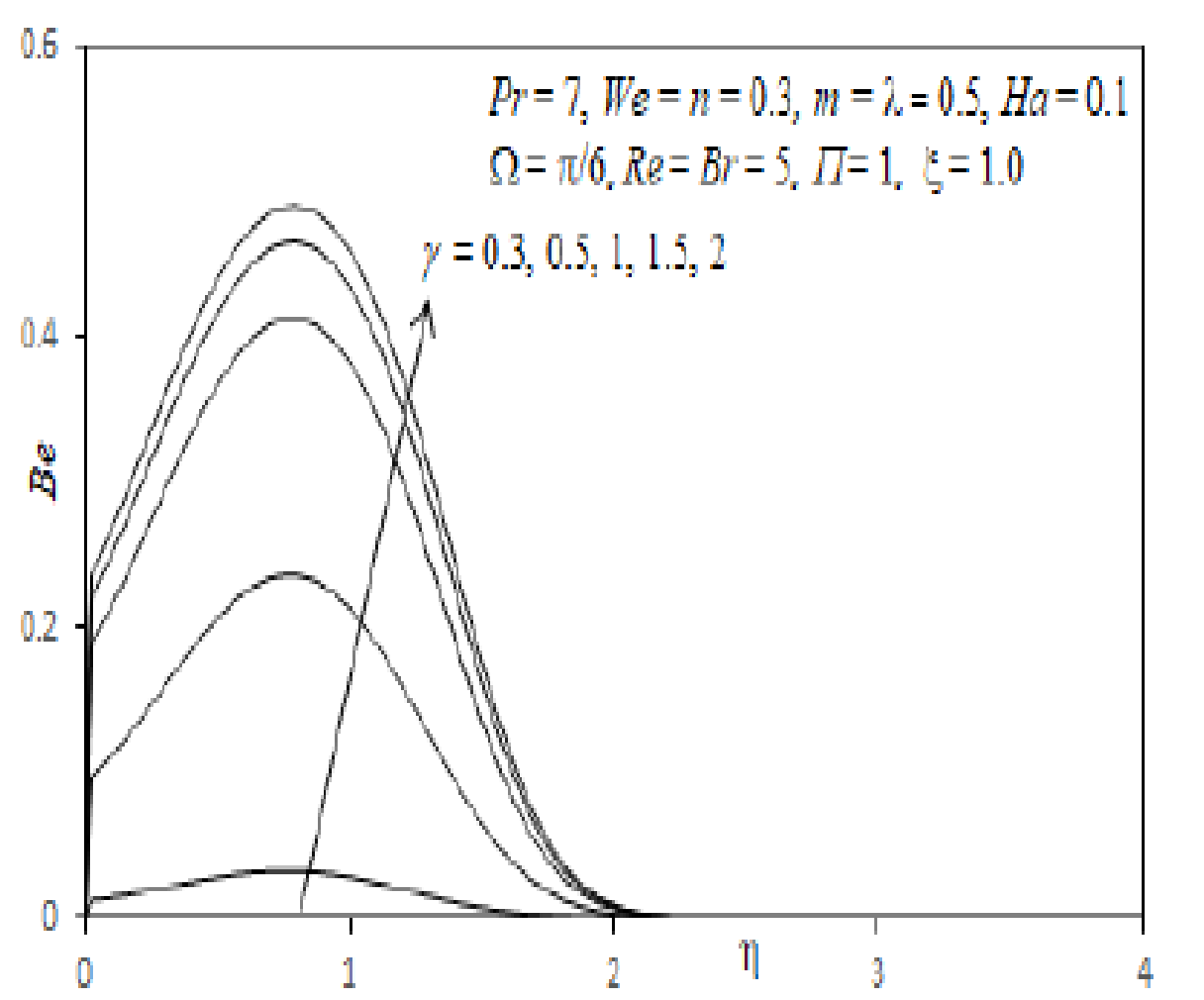

Fig. 22 Intluesce of $\gamma$ on Bejan Number 


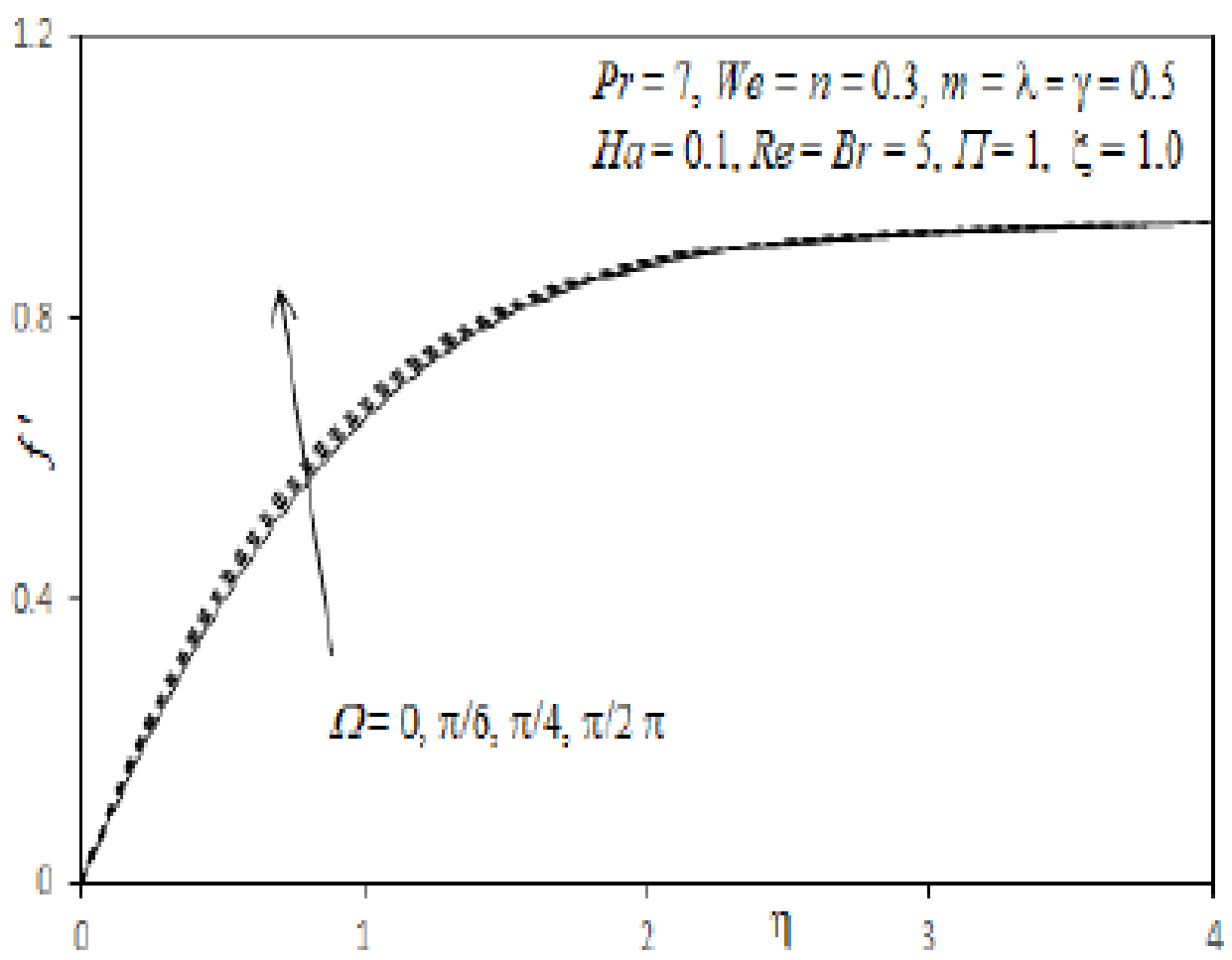

Fig. 23 Influence of $\Omega$ on Velocity Profiles

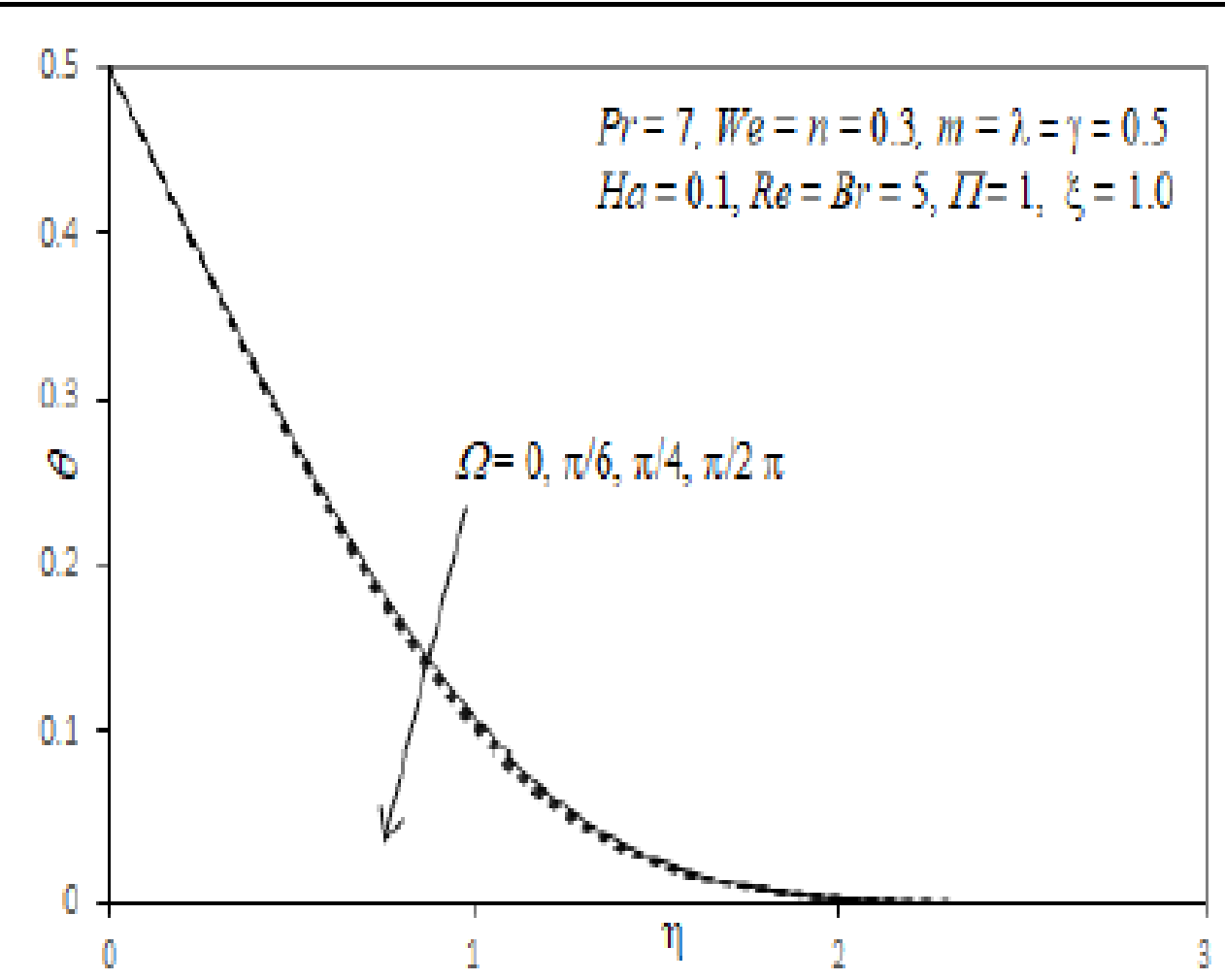

Fig. 24 Influence of $\Omega$ on Temperature Profiles 


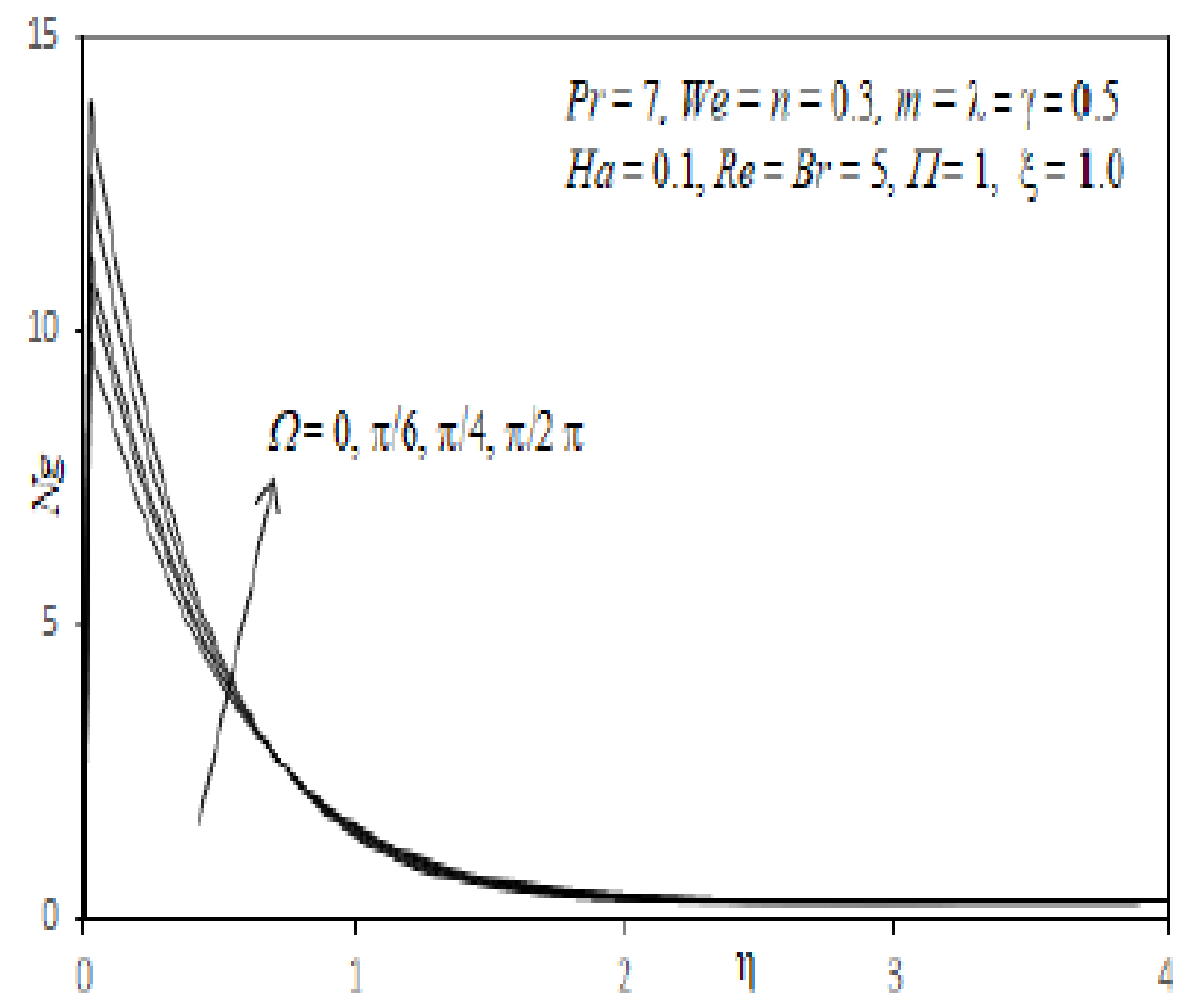

Fig. 25 Influence of $\Omega$ on Entopy

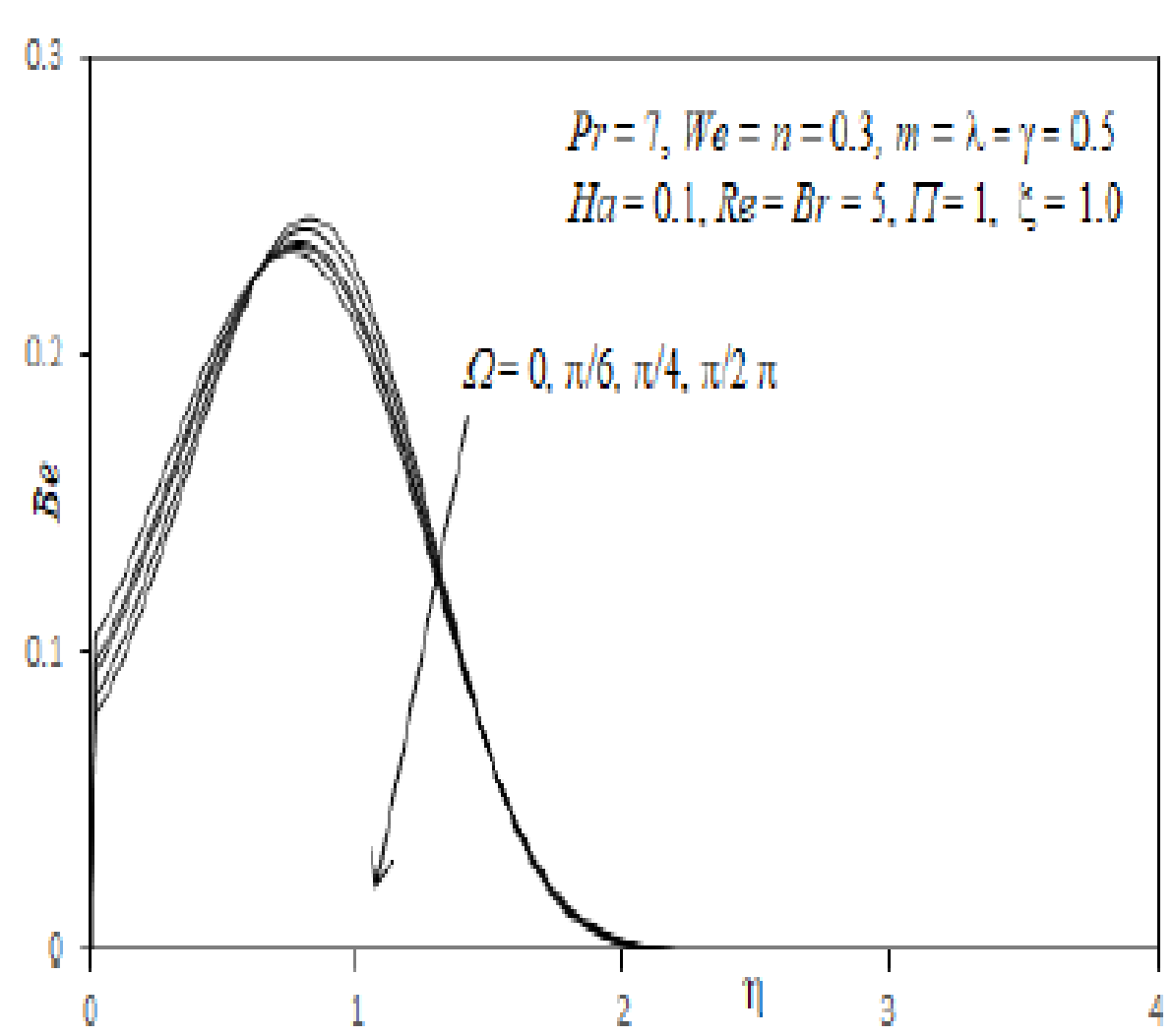

Fig. 26 Influesce of $\Omega$ on Bejan Nunibes 


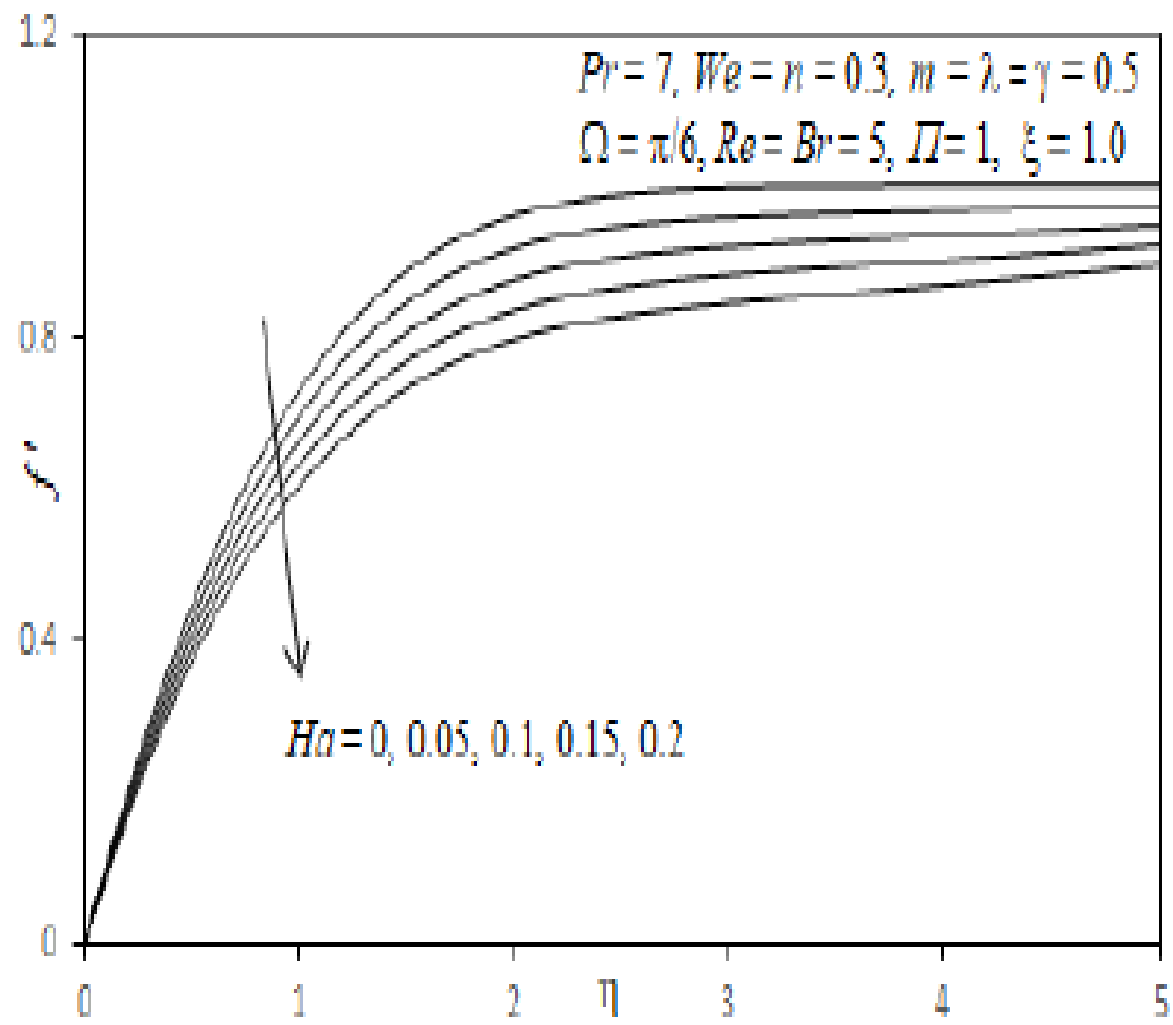

Fig. 27 Influence of $\Omega$ on Velocity Profles

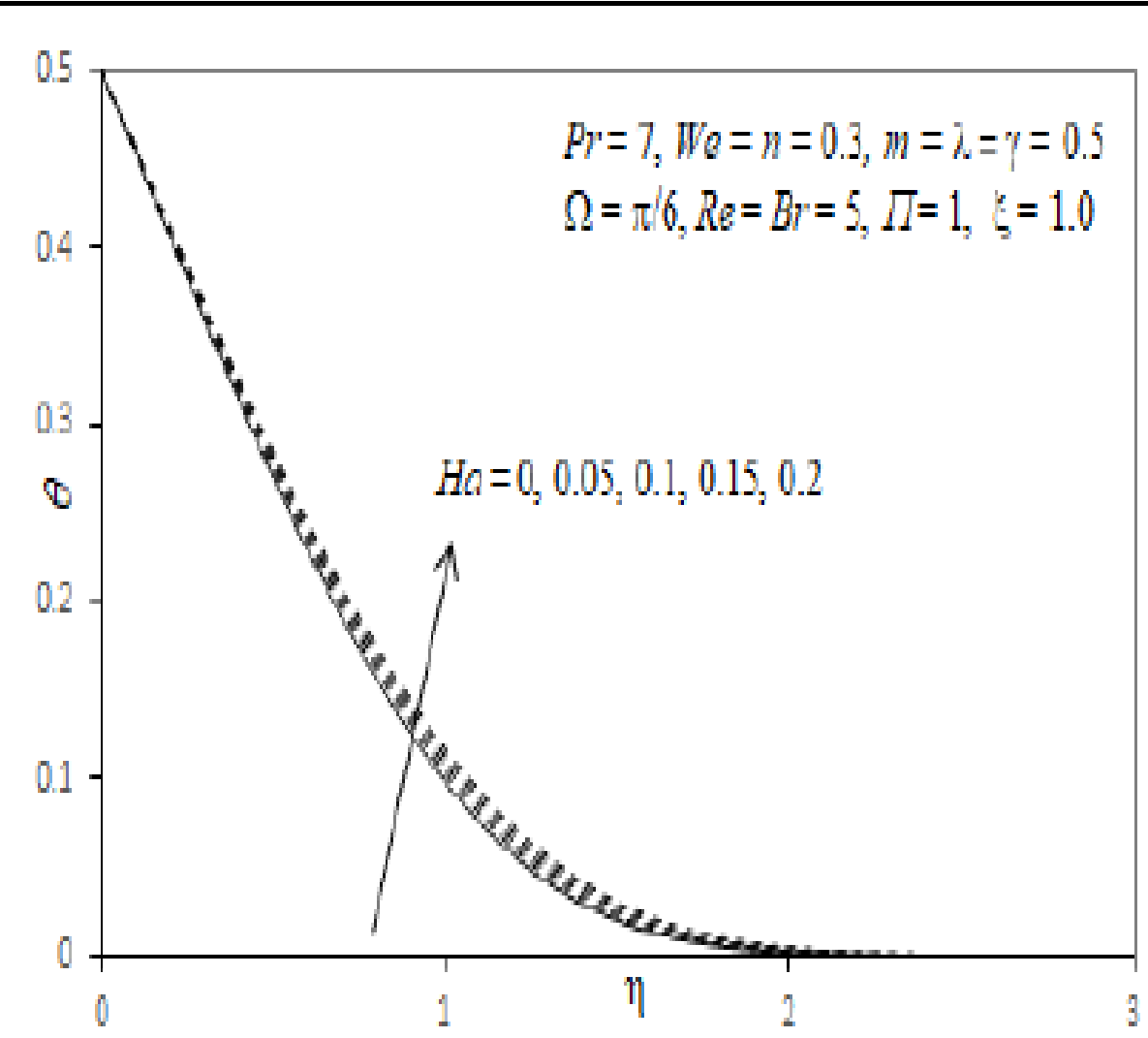

Fig. 28 Influence of $\Omega$ on Tenperature Profiles 


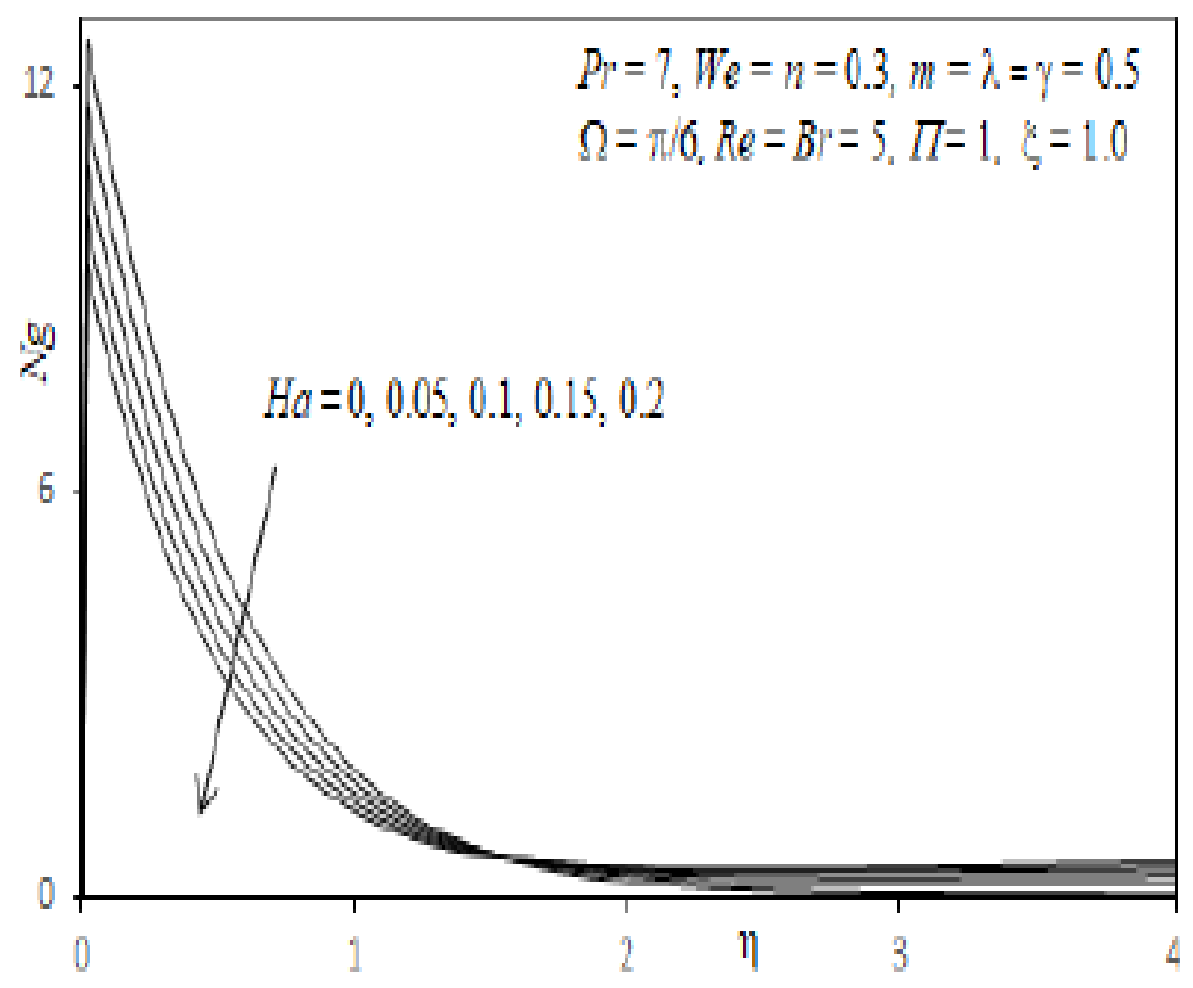

Fig. 29 Influence of $\Omega$ on Entopy

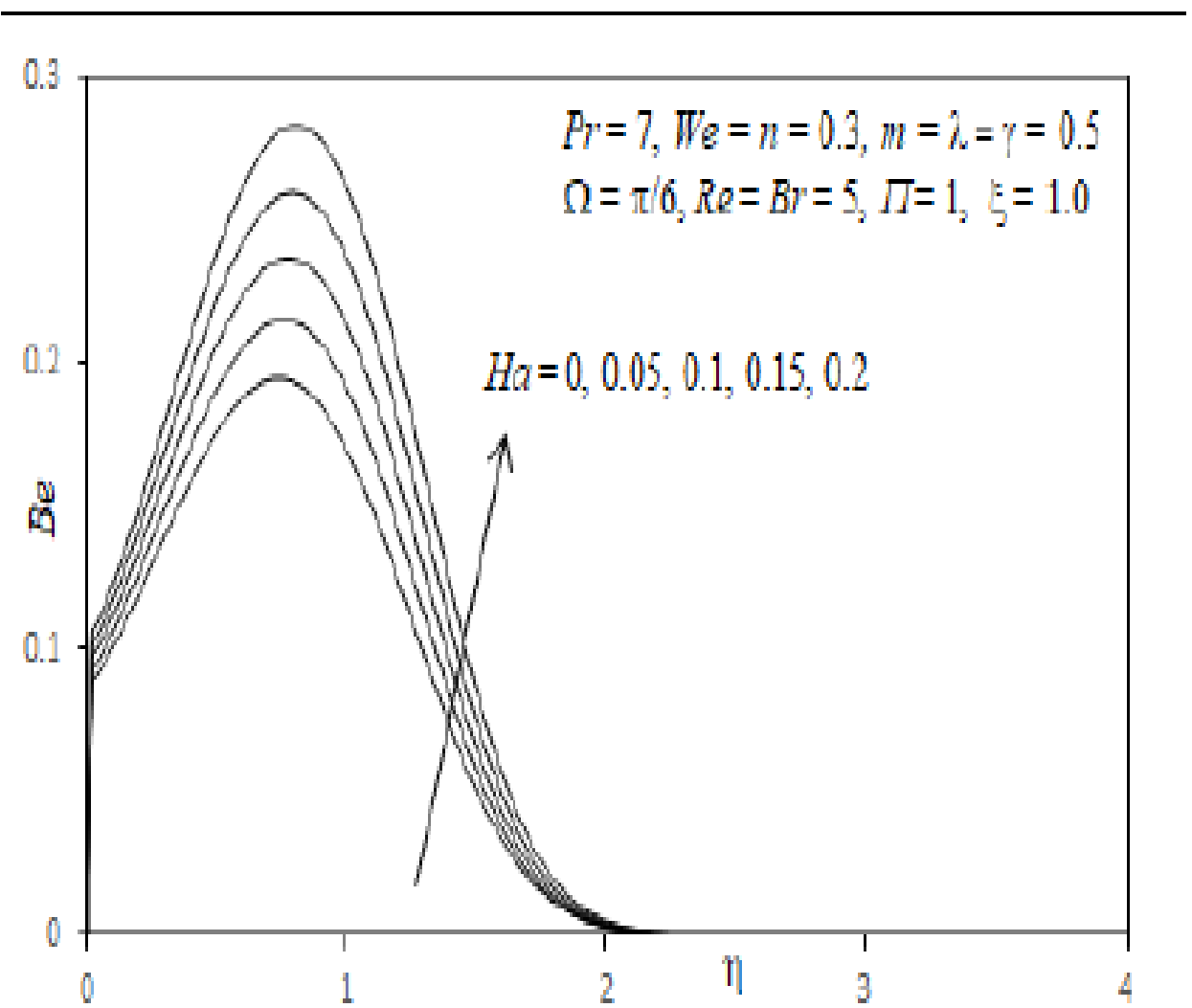

Fig. 30 Influence of $\Omega$ on Bejan Nurnber 


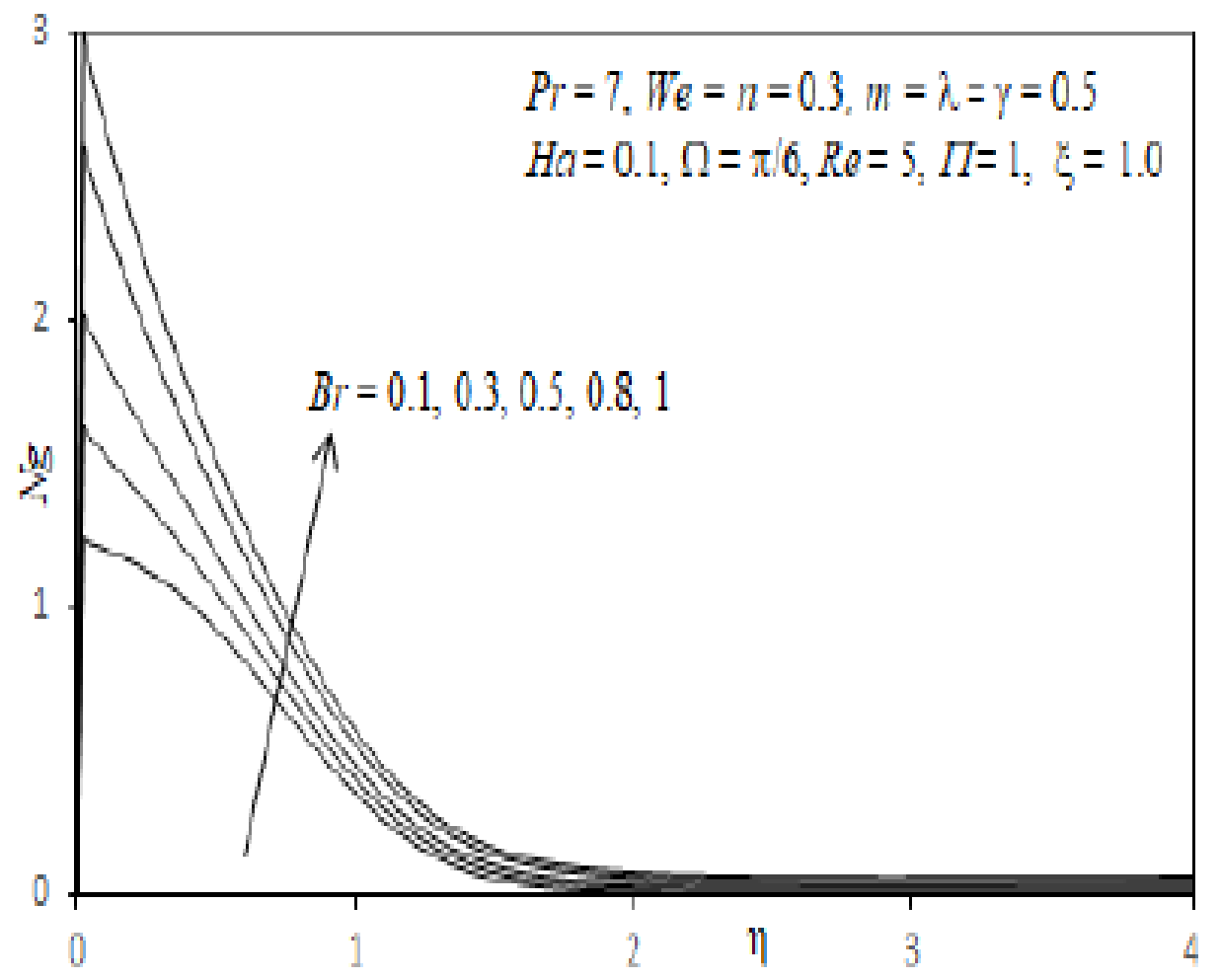

Fig. 31 Influence of $B r$ on Entropy

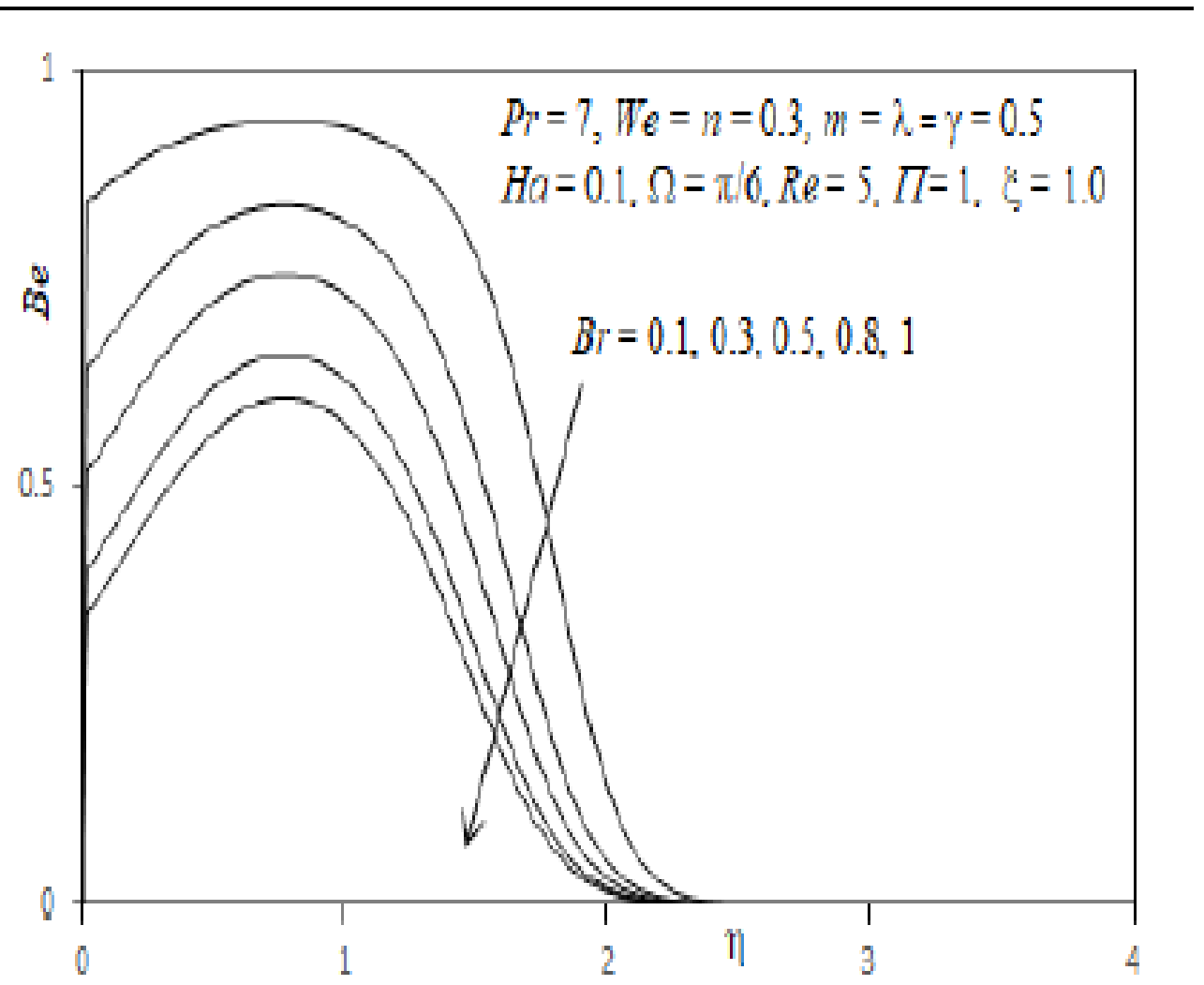

Fig. 32 Influence of $B r$ on Bejan Number 


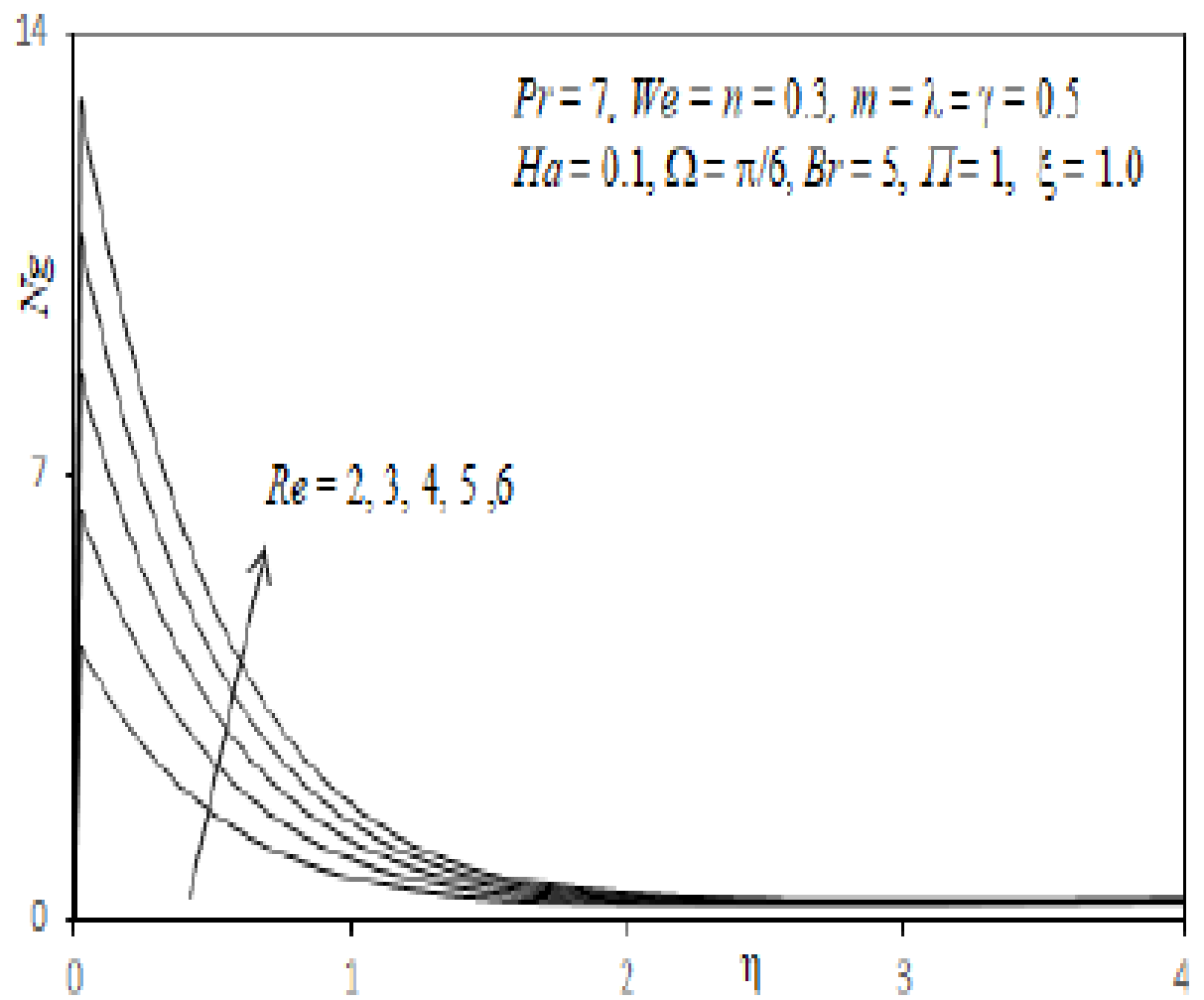

Fig. 33 Infuence of Re on Entropy

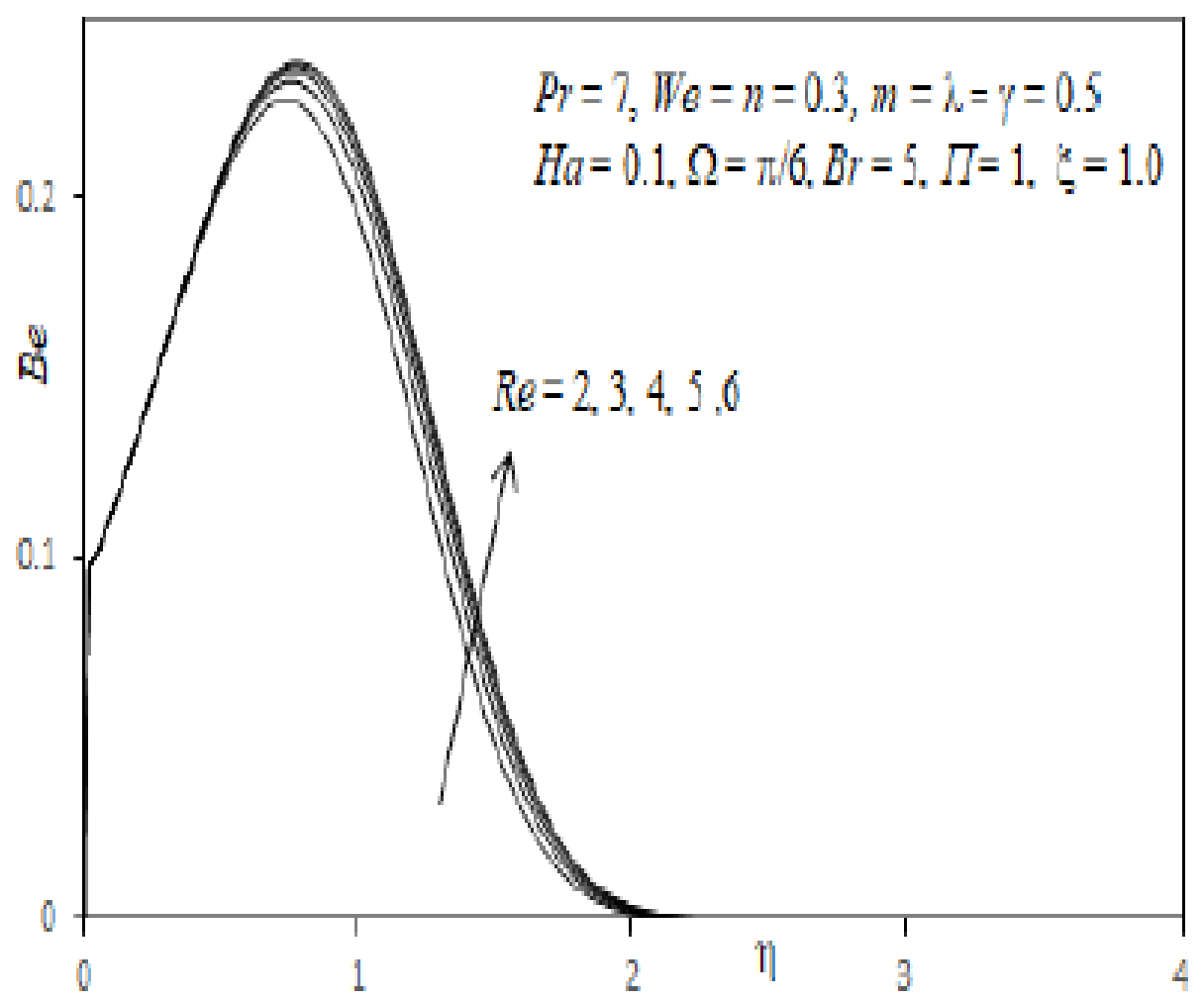

Fig. 34 Influence of Re on Bcian Number 